NBER WORKING PAPER SERIES

\title{
NECKTIES IN THE TROPICS: \\ A MODEL OF INTERNATIONAL TRADE AND CULTURAL DIVERSITY
}

\author{
James E. Rauch \\ Vitor Trindade \\ Working Paper 11890 \\ http://www.nber.org/papers/w11890 \\ NATIONAL BUREAU OF ECONOMIC RESEARCH \\ 1050 Massachusetts Avenue \\ Cambridge, MA 02138 \\ December 2005
}

Rauch is also affiliated with the NBER. Our thanks to Helen and Newton Harrison for initial inspiration, to Doris Bittar, George Lewis, Leslie Stern, Sae Kyung Yu, and especially Dan Hallin for many helpful suggestions, and to Jennifer Poole for excellent research assistance. We also thank participants in the NOITS 7th Annual Workshop, the Workshop on Recent Advances in International Economics (City University of Hong Kong) and seminars at American University of Beirut, Cornell University, the Econometric Society (Summer Meeting), Michigan State University, the Midwest Trade Meetings (University of Kansas), the NBER (ITI Program Meeting, Palo Alto), the University of Michigan, and UCSD. We are responsible for any errors. The views expressed herein are those of the author(s) and do not necessarily reflect the views of the National Bureau of Economic Research.

(C2005 by James E. Rauch and Vitor Trindade. All rights reserved. Short sections of text, not to exceed two paragraphs, may be quoted without explicit permission provided that full credit, including (C) notice, is given to the source. 
Neckties in the Tropics: A Model of International Trade and Cultural Diversity

James E. Rauch and Vitor Trindade

NBER Working Paper No. 11890

December 2005

JEL No. F12, F13, F15, Z10

\begin{abstract}
Some cultural goods, like clothes and films, are consumed socially and are thus characterized by the same consumption network externalities as languages. At the same time, producers of new cultural goods in any one country draw on the stock of ideas generated by previous cultural production in all countries. For such goods, costless trade and communication tend to lead to the dominance of one cultural style, increasing utility in the short run but reducing quality and generating cultural stagnation in the long run. Increasing trade costs while keeping communication costs low may reduce welfare by stimulating production of cultural goods that are "compatible" with the dominant style, thereby capturing consumption network externalities, but that add little to the stock of usable ideas. A reform of cultural policy suggested by our two-country analysis could be to remove import restrictions in the smaller country and replace them with subsidies to the fixed costs of production of new cultural goods in its traditional style.

James E. Rauch

Department of Economics University of California, San Diego

La Jolla, CA 92093-0508

and NBER

jrauch@ucsd.edu

Vitor Trindade

Department of Economics

University of Missouri, Columbia

Columbia, MO 65203

trindadev@missouri.edu
\end{abstract}




\section{Introduction}

In this paper we intend to answer at least three questions. First, why is the market share of U.S. or Anglo-American cultural goods so high in other cultures, and rising? Second, why is cultural trade different from other trade in its welfare implications - or is it? Third, what are the likely effects of policies adopted to protect domestic cultural goods production? Our answers to these questions will lead to specific proposals regarding policy reforms in the area of international trade in cultural goods.

Like others, we will argue that part of the reason for U.S. dominance in cultural goods is the home market effect (Helpman and Krugman 1985, section 10.4). The home market effect results from the interaction of transportation costs with increasing returns to scale in a model of trade in differentiated products. Transportation costs for cultural goods such as film and music are negligible, but as Hoskins et al. (1997) have pointed out, their place is taken by the "cultural discount" that consumers apply to cultural goods from a different culture.

However, we question whether the standard home market effect alone can explain the extent of U.S. dominance in cultural goods. Table 1 shows that in 2002 the median U.S. cinema market share across three large European and two Asian countries was 63 percent and the median ratio of the U.S. to national cinema market share was 2.9. The U.S. cinema market share rivals the U.S. market share of roughly 70 percent (in 1998) in large commercial jet aircraft (Pavcnik 2002), which may be the highest U.S. market share for any well-defined commercial manufactured product. We lack data for the AngloAmerican share of the popular music market, but we suspect that it is also unusually high, though probably not as high as the U.S. film market share (Economist 1998). 
U.S. or Anglo-American dominance could be explained by network externalities in cultural goods consumption. Many writers have recognized the social nature of cultural goods consumption (Cowen 2002, pp. 107-8; Eaton, Pendakur, and Reed 2003). Traditionally these social interactions have taken place within a culture, but falling communication and transportation costs, student exchanges, and most recently internet chat rooms and music file-sharing have increased social interactions across cultures. It is no surprise then that the U.S. cultural market share is increasing, as reflected in the cinema market shares reported in Table 1 . If everyone communicates equally with everyone else the standard of the larger country will tend to take over, whereas there is more possibility for "niche" production to survive if groups are isolated from communicating with each other and have different underlying preferences.

Janeba (2004) sees consumption of cultural goods as an input to production of national "identity," giving rise to special welfare implications of cultural goods trade. In contrast, from a static point of view we treat trade in cultural goods as being no different than trade in any consumption network externality good, such as computer software. We emphasize instead a difference in the dynamic welfare implications of such trade. As in Romer (1990) and Grossman and Helpman (1991), we argue that production of current cultural goods generates ideas that spill over to future cultural goods production. We also make the crucial assumptions that producers of cultural goods have access to the ideas generated by past cultural goods production of all cultures, and that ideas of different cultures are imperfect substitutes.

There can be little doubt that producers of commercially lucrative cultural goods are influenced by ideas from other cultures. This is true of producers in the dominant 
culture as well. For example, the "film noir" style, considered by many to be the most enduring achievement of 1940s Hollywood, was heavily influenced by German expressionist cinema. ${ }^{1}$ George Lucas spent months in Japan in the early 1970s soaking up Japanese films before creating Star Wars, which borrows many key elements directly from the work of the great Japanese director Akira Kurosawa. ${ }^{2}$ In music, it is universally acknowledged that Anglo-American "rock and roll" grew out of "rhythm and blues," a musical style of African-Americans in the U.S. South (see, e.g., Stuessy 1990) that in turn has its roots in West Africa. ${ }^{3}$ The Beatles then led a revolution in rock and roll while under the influence of the European avant-garde, especially the electronic music of Karlheinz Stockhausen. ${ }^{4}$

The imperfect substitutability of ideas from different cultures in production of new cultural goods is a more controversial assumption, and it has the crucial implication that the ideas that spill over from the cultural goods production of the subordinate culture(s) are more valuable because they are more scarce. Cultural scholars categorize film and music by national or cultural/linguistic "schools" or genres, each of which has a set of recognizable characteristics. ${ }^{5}$ It stands to reason, then, that a producer of new film

\footnotetext{
${ }^{1}$ Hirsch (1981, p. 53) writes, "The cinematic origins of film noir can be traced to the German Expressionist films of the late 1910s and twenties". Double Indemnity (1944) is one of the signature Hollywood films in this style.

${ }^{2}$ Baxter (1999, p. 73) states, "Lucas loved the formalized sword-duels of Kurosawa's historical films.....No less attractive were his themes: loyalty to a lord; honor; mutual respect among warriors; fidelity to bushido, the samurai code."

${ }^{3}$ Evans (2002, pp. 23-24) describes the influences of African music on the harmony, instrumentation, and even subject matter of rhythm and blues songs.

${ }^{4}$ Everett (1999, p. 10) writes that Paul McCartney "introduced the Beatles to the worlds of Stockhausen and Bach, leading to a revolution in the expressive capacity of mainstream rock music." Stockhausen appears in the crowd on the celebrated cover of Sgt. Pepper's Lonely Hearts Club Band.

${ }^{5}$ For example, Thomas (1985), writing about "Indian popular cinema," states, "What seems to emerge in Hindi cinema is an emphasis on emotion and spectacle rather than tight narrative, on how things will happen rather than what will happen next, on a succession of modes rather than linear denouement, on familiarity and repeated viewings rather than 'originality' and novelty, on a moral disordering to be (temporarily) resolved rather than an enigma to be solved." Slonimsky (1997, p. 29) states of "Arab
} 
or music will get ideas from watching, listening to, and studying the film or music of other cultures that are qualitatively different from the ideas he absorbs from the film or music of his own culture. ${ }^{6}$

At first glance our argument suggests that, by preserving cultural diversity, protection of cultural goods production can generate dynamic welfare gains that offset the static welfare losses it causes. ${ }^{7}$ To understand the likely long-term effects of protection, however, we turn to a cultural good for which the rest of the world does not fear U.S. or Western dominance, but quite the opposite: clothing. Clothing is not only an integral part of culture but also a consumption network externality good par excellence: people are more comfortable when they are dressed like those around them. Perhaps as a result, Western clothing appears to have achieved a market share outside of the West comparable to the U.S. cinema market share, despite its occasional inappropriateness (as millions of necktie-wearing tropical businessmen and office workers will attest). At the same time, clothing was the lead industry in import-substituting industrialization throughout the world, and now the Western share of clothing production is far smaller than the Western share of clothing style.

The example of clothing shows that the long-term effect of protection is not likely to be preservation of cultural diversity but rather imitation of the goods produced by the dominant culture, as producers in other cultures respond to the demand created by

music" that "The traditional music of Arab nations of the Mediterranean and Persian Gulf basins differs so greatly from the nature of Western music that transcription with any degree of fidelity into Western notation is fraught with difficulties....The melodies themselves register to a Western-trained ear as progressions of quarter tones, semitones, and other divisions of a whole tone."

${ }^{6}$ In our model, we will assume that the same set of ideas processed through an Indian or Arab "sensibility" yields Indian film or Arab music rather than U.S. film or music. We argue that the same cannot be said of a spreadsheet program, for example, which will be no more different from other spreadsheet programs than if it had been designed by U.S. software producers.

${ }^{7}$ Ottaviano and Peri (2004) find evidence that cultural diversity makes U.S. cities more productive. 
consumption network externalities. An excellent example is provided by the experience of Argentina in the 1990s. Under the Menem regime, heavy state subsidies were provided to domestic film production. Content was deregulated but participation of domestic television stations was encouraged to insure commercial viability of output. The result was the production of films such as Comodines (Cops), billed as "the first Hollywood-style movie spoken in Spanish," and a smash hit in Argentina (Falicov 2000, p. 330).

Ironically, the Argentine experience also points towards the policy recommendations generated by our model. As in the 1990s under Menem, in the 1980s under Alfonsín Argentina provided state subsidies to domestic film production, but projects were chosen "with an international film festival audience in mind, rather than a domestic one" (Falicov 2000, p. 334). This maximized the spillover of ideas from Argentine film output while allowing Argentine consumers to enjoy the consumption network externalities from U.S. films. What made this policy package unsustainable, of course, was that the main beneficiaries were not the Argentine taxpayers who were financing the subsidies. It follows that if this kind of policy package is to be sustained, the foreigners who reap most of the benefits could have to bear more of the subsidy burden.

To support the intuition presented here and generate additional results, in the next section we will develop a model of international trade in differentiated products subject to consumption network externalities. The static part of our model should apply equally well to non-cultural goods subject to such externalities, such as computer software. We believe that the dynamic part and the policy implications that flow from it only apply to 
trade in cultural goods, however. In section III we derive both positive and normative results for our basic model. We add the possibility of imitative cultural goods production in section IV. In our concluding section we explore the lessons that our model and results provide for cultural policy.

\section{The Model}

Our model builds upon the well-known home market effect model of Helpman and Krugman (1985, section 10.4), and we will adopt their notation where convenient. There are two industries, one producing a differentiated product and the other a homogeneous product. We will call the differentiated product industry the cultural goods industry. We assume Cobb-Douglas utility for the two goods, yielding constant expenditure shares, and we will let the sub-utility for cultural goods take the standard CES form. There are two countries, home and foreign. We distinguish all foreign consumption with an asterisk, and all foreign production with a tilde (thus, for example, $C^{*}$ will denote foreign consumption of the home cultural good, while $\widetilde{C}$ is home consumption of the foreign cultural good). Production technology is identical across countries, but the cultures of each country are distinct. There is one factor of production, which we shall call labor.

The homogeneous good is costlessly tradable and both countries produce it when there is trade. It is produced under constant returns to scale and perfect competition, which ensures that the wage rate $w$ will be the same in both countries. Letting the homogeneous good be the numeraire and assuming (without loss of generality) that each unit requires one unit of labor to produce, we have $w=w^{*}=1$ in equilibrium. Cultural 
goods are produced under increasing returns to scale, with a fixed cost and constant marginal cost, in a monopolistic competition setting.

Our first change to the Helpman-Krugman model is that we assume that cultural goods are costlessly transportable, but they are subject to a "cultural discount" by domestic consumers. This change proves to be only notational, i.e., the cultural discount in our model is equivalent to the "ice" transportation cost in the Helpman-Krugman model. Our second change is to assume that the utility from consuming cultural goods is augmented by consumption network externalities, which we will specify below. Our third change is to assume that the utility from consumption of cultural goods is also augmented by the quality of cultural goods production, which in turn is a function of past cultural goods production through spillover of ideas.

These three changes are incorporated into the utility function of the representative home country consumer:

$$
\begin{aligned}
& u_{\tau}=C_{y \tau}^{1-\alpha}\left\{Q_{\tau}\left[\sum_{i=1}^{n_{\tau}}\left(H_{\tau} C_{i \tau}\right)^{\rho}+\sum_{j=1}^{\tilde{n}_{\tau}}\left(\delta \widetilde{H}_{\tau} \widetilde{C}_{j \tau}\right)^{\rho}\right]^{1 / \rho}\right\}^{\alpha}, \\
& U_{t}=\sum_{\tau=t}^{\infty} \frac{u_{\tau}}{(1+r)^{\tau}},
\end{aligned}
$$

where $\tau$ indexes time; $C_{y}$ is the consumption of the homogeneous good by the representative home country consumer; $C_{i}$ and $\widetilde{C}_{j}$ are the consumptions by the home consumer of varieties $i$ and $j$ of the home and foreign cultural good, respectively; $\delta$ is the cultural discount rate, where we assume that $0<\delta<1 ; H$ and $\widetilde{H}$ measure the consumption network externalities enjoyed by consuming home and foreign cultural goods, respectively; $n$ and $\tilde{n}$ are the number of varieties of home and foreign cultural 
good that are produced, respectively; $Q$ is the quality of cultural goods production; $r$ is the time discount rate; $0<\rho<1$; and $0<\alpha<1$. In equation (1), $u_{\tau}$ is the instantaneous utility at time $\tau$, and $U_{t}$ is total utility, discounted to time $t$.

The utility of the representative foreign country consumer is symmetric to equation (1) and can be written as follows:

$$
\begin{aligned}
& u_{\tau}^{*}=\left(C_{y \tau}^{*}\right)^{1-\alpha}\left\{Q_{\tau}\left[\sum_{i=1}^{n_{\tau}}\left(\delta H_{\tau}^{*} C_{i \tau}^{*}\right)^{\rho}+\sum_{j=1}^{\tilde{n}_{\tau}}\left(\widetilde{H}_{\tau}^{*} \widetilde{C}_{j \tau}^{*}\right)^{\rho}\right]^{1 / \rho}\right\}^{\alpha}, \\
& U_{t}^{*}=\sum_{\tau=t}^{\infty} \frac{u_{\tau}^{*}}{(1+r)^{\tau}},
\end{aligned}
$$

where the notation is in all respects analogous to equation (1). Note that the assumption of equal cultural discounting by home and foreign consumers is made for notational simplicity, and does not qualitatively affect our results.

Equations (1) and (2) incorporate the assumption that the quality of all cultural goods production is the same across the two countries, implying that the ideas generated by production of past cultural goods have the same spillover to both home and foreign producers. Thus, the model works as if all cultural goods producers attend the same international film and music festivals, making the ideas from past cultural production a global public good. We could easily let each country's producers give more weight to the ideas generated by past production in their own country without qualitatively changing our results, but we maintain symmetry for simplicity.

For the quality of current period production of cultural goods we specifically assume:

$$
Q_{\tau}=Q\left(n_{\tau-1}, \widetilde{n}_{\tau-1}\right) .
$$


Equation (3) incorporates two important additional assumptions. First, ideas generated by past production of home and foreign varieties of cultural goods are imperfect substitutes in production of current cultural good quality, as we argued in our introduction. We assume this implies that the marginal product of an additional past variety of a country is increasing with the relative scarcity of that country's past varieties. Second, current cultural goods quality depends only on varieties produced in the immediately preceding period; there is no accumulation of ideas that would cause cultural goods quality to increase over time. ${ }^{8}$ This means that there is no state variable in the model, and we can drop the time subscript to simplify notation.

Given the symmetry with which both home and foreign varieties of cultural good enter the utility function of the representative consumer for the home or foreign country, demand by either consumer will be identical across home or foreign varieties. This allows us to define consumption network externalities compactly, thereby completing the specification of our model:

$$
\begin{aligned}
& H \equiv H\left(n D+m D^{*}\right), \widetilde{H} \equiv H\left(\widetilde{n} \widetilde{D}+\widetilde{n} \widetilde{D}^{*}\right), \\
& H^{*} \equiv H\left(m D+n D^{*}\right), \widetilde{H}^{*} \equiv H\left(\widetilde{\eta} \widetilde{D}+\widetilde{n} \widetilde{D}^{*}\right),
\end{aligned}
$$

where $D$ is aggregate demand for a given variety of cultural good, with an asterisk denoting demand by foreign consumers and a tilde denoting demand for foreign varieties. In equation (4), $\gamma$ is a parameter such that $0<\gamma<1$, and $H($.) is an increasing function of its argument, with the normalization $H(0)=1$. There are two key features of our

\footnotetext{
${ }^{8}$ Thus, cultural goods producers in our model are only influenced by "fresh ideas." In reality, current cultural goods producers may also get ideas from archived film and music, for example, nearly all of which loses relevance with time. Allowing for less than 100 percent depreciation of cultural ideas after two periods would mean that the impact on future cultural goods quality of changes in current numbers of varieties of cultural goods being produced would unfold more slowly.
} 
specification. First, consumption network externalities are stronger within a country than across countries, with the extent of cross-country externalities determined by $\gamma$, a parameter that we can think of as measuring "globalization." Second, externalities for any variety depend not only on consumption of that individual variety but also on consumption of all "compatible" varieties, i.e., varieties produced within the same culture.

This second feature may be controversial. We argue that a major reason why film is socially consumed is that people like to discuss the films they see. Discussion will often involve comparison with other, similar films, so enjoyment of any given film is enhanced by the extent to which similar films have been seen by the participants in the discussion. Music needs to be compatible to be programmed by DJs at events and on the radio and for dance music at parties, so again, demand for any given piece of music is enhanced by the extent to which similar music is consumed by others. In other words, compatible films and music create a social context within which a given film or piece of music is consumed, without which it is less enjoyable. Obviously, the symmetry across all varieties in our consumption network externality specification is unrealistic: externalities will be stronger within than across genres (action-adventure versus romantic comedy, country-western versus rock), and stronger for the variety in question than for other compatible varieties. Incorporating these complications would not qualitatively affect our results, provided that each individual producer is still too small to affect the sum of consumption network externalities for its variety of cultural good. 


\section{Model Solution and Results}

It is easy to show that we obtain the standard CES demands for cultural goods, which are therefore given by:

$$
\begin{aligned}
& D=\frac{p^{-\sigma} H^{\sigma-1}}{n p^{1-\sigma} H^{\sigma-1}+\widetilde{n} p^{1-\sigma}(\delta \tilde{H})^{\sigma-1}} \alpha L, \\
& \widetilde{D}=\frac{p^{-\sigma}(\delta \tilde{H})^{\sigma-1}}{n p^{1-\sigma} H^{\sigma-1}+\widetilde{n} p^{1-\sigma}(\delta \tilde{H})^{\sigma-1}} \alpha L, \\
& D^{*}=\frac{(p T)^{-\sigma}\left(\delta H^{*}\right)^{\sigma-1}}{n(p T)^{1-\sigma}\left(\delta H^{*}\right)^{\sigma-1}+\widetilde{n} p^{1-\sigma} \widetilde{H}^{* \sigma-1}} \alpha L^{*}, \\
& \widetilde{D}^{*}=\frac{p^{-\sigma} \widetilde{H}^{* \sigma-1}}{n(p T)^{1-\sigma}\left(\delta H^{*}\right)^{\sigma-1}+\widetilde{n} p^{1-\sigma} \widetilde{H}^{* \sigma-1}} \alpha L^{*} .
\end{aligned}
$$

Here $\sigma \equiv 1 /(1-\rho)$ is the elasticity of substitution between any two varieties of the cultural good, $p$ is the price of any variety in either country in monopolistic competition equilibrium, $T$ equals one plus the tariff rate that the foreign country may impose on cultural goods, and $L$ and $L^{*}$ are both the home and foreign country labor forces, respectively, and the home and foreign country consumer incomes (recall that $w=\widetilde{w}=1$ ). For simplicity we have assumed that the government spends all tariff revenue on the homogeneous product. In monopolistic competition equilibrium, $p$ will be fixed by the constant markup and the constant wage (which determines a constant marginal cost). Note that cultural goods quality $Q$ drops out of the demand for cultural goods. This occurs because the Cobb-Douglas specification of the utility function makes $Q^{\alpha}$ a shifter of the entire level of utility. Therefore, $Q^{\alpha}$ is only used when we discuss the welfare implications of the model. 
As in the Helpman-Krugman model, the output of any variety in monopolistic competition is fixed and equal across countries. Denoting this output by $x$, we have two market clearing conditions:

$$
\begin{aligned}
& D+D^{*}=x, \\
& \widetilde{D}+\widetilde{D}^{*}=x .
\end{aligned}
$$

Equations (5) and (6) treat demand and supply for cultural goods such as film and music analogously to demand and supply for cultural goods such as clothing, in that the price paid by consumers and marginal cost incurred by producers is positive. This is not a problem if film is consumed by purchasing DVDs and music is consumed by purchasing CDs, but would seem inapplicable to music heard on the radio, for example. We argue nevertheless that the "price" to consumers of listening to music on the radio is still positive provided that consumers have a positive opportunity cost of time, so that demand measured in listening time is still described by equations analogous to equations (5). Moreover, revenue received by producers is proportional to the number of listeners through royalty agreements, and producers must still cover their fixed costs of production, to which $x$ is proportional. In short, we argue that equilibrium conditions that are qualitatively equivalent to equations (6) (i.e., yield the same economic behavior) would emerge from a fully specified model of any cultural goods market in which intellectual property rights are fully protected, as we shall assume throughout this paper.

Equations (5) and (6) give us six equations in the six unknowns: $n, \tilde{n}, D, \widetilde{D}, D^{*}$, and $\widetilde{D}^{*}$. If the function $H$ were identically one, the model would reduce to the HelpmanKrugman model. Indeed, we will need to place some restrictions on the function $H$ in order to ensure that our model behaves enough like the Helpman-Krugman model to 
admit a unique interior equilibrium, i.e., an equilibrium where there is positive production of cultural goods in both countries and where the corner solutions that often occur in models with externalities are ruled out. ${ }^{9}$ In such an equilibrium, not only do both market clearing equations (6) hold but also $D+D^{*}>x$ when the model is evaluated at $n=0$ and $\widetilde{D}+\widetilde{D}^{*}>x$ when $\tilde{n}=0$, so that corner equilibria are eliminated by entry.

The first restriction on the function $H$ needed to ensure the existence of an interior equilibrium is that it is bounded from above by $1 / \delta$. To see why, combine the two market-clearing conditions to obtain:

$$
D-\widetilde{D}=\widetilde{D}^{*}-D^{*} \text {. }
$$

Substituting equations (5) for the demands in the equation above and eliminating $p$ and $\alpha$ yields:

$$
\frac{\left[H^{\sigma-1}-(\delta \tilde{H})^{\sigma-1}\right] L}{n H^{\sigma-1}+\widetilde{n}(\delta \tilde{H})^{\sigma-1}}=\frac{\left[\widetilde{H}^{* \sigma-1}-\left(\delta H^{*}\right)^{\sigma-1}\right] L^{*}}{n\left(\delta H^{*}\right)^{\sigma-1}+\widetilde{n} \widetilde{H}^{* \sigma-1}} .
$$

Now suppose that $\tilde{n}$ approaches zero, so that $\tilde{H}, \widetilde{H}^{*} \rightarrow 1$. Then $\widetilde{D}^{*}-D^{*}$ (the right-hand side of the equation above) approaches a positive term times $1-\left(\delta H^{*}\right)^{\sigma-1}$. Therefore, we need $H^{*}<1 / \delta$, since $D-D^{*}$ (the left-hand side of the equation) is certainly positive. The intuition for this restriction is that if positive production of cultural goods is going to be possible in the foreign (small) country, the consumption network externality cannot increase the desirability of the home cultural goods sufficiently to more than offset the cultural discount.

The second restriction we need to place on the function $H$ is that its derivative not

\footnotetext{
${ }^{9}$ Multiple specialized equilibria ("standards") are a common feature of models with network externalities (see, e.g., Church and Gandal 1992).
} 
be too large, a restriction made more precise in the proofs of the various propositions below. To see the need for this restriction, consider the condition $D+D^{*}>x$ when the model is evaluated at $n=0$, which rules out the existence of a corner equilibrium in which only the foreign country produces cultural goods (analysis of the other corner equilibrium is symmetric). As entry occurs and $n$ rises, $H$ and $H^{*}$ increase, tending to raise $D$ and $D^{*}$ by equations (5) and stimulate more entry, driving the economy to the opposite corner equilibrium. Working against this self-reinforcing mechanism is the fact that each additional home variety diverts home demand from other home varieties more than it diverts foreign demand from foreign varieties, because foreign spending on home varieties is reduced by the cultural discount. Thus the increase in consumption network externalities that occurs as the number of home varieties consumed increases must be dominated by the tendency to diversify consumption induced by the cultural discount, requiring the derivative of $H$ to be sufficiently small. ${ }^{10}$

With these restrictions on the function $H$ in place, we can begin to simplify the system of six equations in six unknowns given by (5) and (6). First, we use the accounting identities that consumer spending on home and foreign cultural goods equals their total expenditures on cultural goods:

$$
\begin{aligned}
& n p D+\widetilde{n} p \widetilde{D}=\alpha L \\
& n p T(x-D)+\widetilde{n} p(x-\widetilde{D})=\alpha L^{*},
\end{aligned}
$$

where we have substituted the market clearing conditions (6) into equation (8). Then equations (7) and (8) can be "solved" for $n$ and $\tilde{n}$ :

\footnotetext{
${ }^{10}$ In numerical simulations of our model we used the function $H(a)=(b+a / \delta) /(b+a)$, which allows us to make the derivative of $H$ as small as necessary by increasing $b$.
} 


$$
\begin{gathered}
n=\frac{\alpha\left(L x-\widetilde{D}\left(L+L^{*}\right)\right) / p}{D \widetilde{D}(T-1)+x(D-T \widetilde{D})} \\
\widetilde{n}=\frac{\alpha\left(D\left(T L+L^{*}\right)-T L x\right) / p}{D \widetilde{D}(T-1)+x(D-T \widetilde{D})} .
\end{gathered}
$$

Equations (9) are not true solutions for $n$ and $\tilde{n}$, since there are endogenous variables on the right-hand sides. However, they do provide a substitution rule for the numbers of varieties produced in the home and foreign countries. Since the market clearing conditions (6) further allow the substitution for $D^{*}$ and $\widetilde{D}^{*}$ everywhere, we are left with only two endogenous variables, namely home demands for the representative home and foreign varieties, $D$ and $\widetilde{D}$. To close the analysis, we must obtain a system of two equations in those two variables. We do so by performing the substitutions described above into the demand equations (5), which after some simplification yields:

$$
\begin{aligned}
& \widetilde{D}=D\left(\frac{\delta \tilde{H}}{H}\right)^{\sigma-1} \equiv D A(D, \widetilde{D}, y, z, \gamma), \\
& x-\widetilde{D}=(x-D) T^{\sigma}\left(\frac{\widetilde{H}^{*}}{\delta H^{*}}\right)^{\sigma-1} \equiv(x-D) T^{\sigma} B(D, \widetilde{D}, y, z, \gamma),
\end{aligned}
$$

where all substitutions are implicit inside the arguments of the $H$ functions, and we have introduced the following re-parameterizations of the country sizes: $y \equiv L+L^{*}$, $z \equiv \frac{L}{L+L^{*}}$. Thus $y$ denotes the size of the world, whereas $z$ denotes the share of the larger (home) country, so that $1 / 2 \leq z<1$. We have also defined the functions $A(D, \widetilde{D}, y, z, \gamma) \equiv(\delta \widetilde{H} / H)^{\sigma-1}$ and $B(D, \widetilde{D}, y, z, \gamma) \equiv\left(\widetilde{H}^{*} / \delta H^{*}\right)^{\sigma-1}$ for future reference. 
In the Helpman-Krugman model, if the relative size of the home country $z$ is sufficiently great production of varieties in the foreign country goes to zero, so that equilibrium with positive numbers of varieties produced in both countries only obtains for a restricted range of $z$. Our first proposition shows that such an upper bound for the relative home country size also exists in our model, and, more importantly, that this upper bound decreases with $\gamma$, implying that globalization causes cultural goods production to disappear from increasingly large foreign countries. Intuitively, increases in relative home country size and increased globalization both reduce demand for the cultural goods of the foreign (smaller) country relative to the home country, so increasing $\gamma$ decreases the relative home country size necessary to eliminate foreign cultural goods production entirely.

Proposition 1: Given sufficiently strong cultural discounting (low $\delta$ ), a non-prohibitive tariff (finite T), and an upper bound on consumption network externalities $H$ that is independent of $\delta$, there exists a "region of cultural diversity" for the home (larger) country share of the world labor force. Specifically, there exists a unique $z_{H}(\gamma)$ such that $n, \tilde{n}>0$ for $1 / 2 \leq z<z_{H}(\gamma)$ and $n>0, \tilde{n}=0$ for $z \geq z_{H}(\gamma)$. Moreover, $z_{H}{ }^{\prime}(\gamma)<0$.

All propositions are proved in the Appendix. ${ }^{11}$ The region of cultural diversity in $(z, \gamma)$ space is shown in Figure 1, where the dashed line and notations $z_{H 1}(\gamma)$ and $Z_{H 2}(\gamma)$ should be ignored for now.

Our next proposition states that within the region of cultural diversity a solution for our model exists and is unique.

Proposition 2: Given $1 / 2 \leq z<z_{H}(\gamma)$, there exist unique positive values of $D, \widetilde{D}, D^{*}$, $\widetilde{D}^{*}, n$, and $\tilde{n}$ that solve equations (6), (9), (10), and (11).

\footnotetext{
${ }^{11}$ Since the proof of Proposition 1 uses the potential to make $\delta$ arbitrarily small, we need a bound on $H$ other than $1 / \delta$. This is a technical rather than a substantive assumption and is not needed (or used) in any of our numerical simulations cited below.
} 
The basis for this proposition can be found in Figure 2, which shows equations (10) and (11), labeled "home consumption schedule" and "foreign consumption schedule," respectively. The figure depicts a square of side $x$, its horizontal edge representing the total production of a typical home variety, and its vertical edge representing the total production of the typical foreign variety. Home consumptions $D$ and $\widetilde{D}$ are measured from the origin $O$ and therefore foreign consumptions $D^{*}=x-D$ and $\widetilde{D}^{*}=x-\widetilde{D}$ are measured from the origin $O^{*}$. A point in the figure therefore represents the allocation of the typical home variety and of the typical foreign variety between the home and the foreign representative consumers. The restrictions $H<$ $1 / \delta$ and $H^{\prime}$ not too large ensure that the slope of the home (foreign) consumption schedule is less (greater) than one and that the schedules are close to straight lines, because any curvature must come from changes in the $H$ functions with respect to their arguments.

We are now ready for comparative static analysis of the interior equilibrium of our model. We first show that the standard home market effect obtains in our model. This is important because we argued in our introduction that increased globalization reinforces the home market effect in the presence of consumption network externalities. From equations (9), we can write the ratio between $\tilde{n}$ and $n$ as follows:

$$
R(z, \gamma, T) \equiv \frac{\widetilde{n}}{n}=\frac{D\left(T L+L^{*}\right)-T L x}{L x-\widetilde{D}\left(L+L^{*}\right)}=\frac{D(T z+1-z)-T z x}{z x-\widetilde{D}}
$$

It is then straightforward to show that this ratio decreases with the home country share of the world labor force. 
Proposition 3: Given $1 / 2 \leq z<z_{H}(\gamma)$, we obtain $\partial R(z, \gamma, T) / \partial z<0$, the home market effect.

We can now go on to state a proposition that the ratio of foreign to home varieties of cultural goods produced in equilibrium decreases as globalization increases. However, unlike Proposition 1 showing that increased globalization causes cultural goods production to disappear from increasingly large countries, we can only prove our proposition on $R$ if there is no protection of foreign cultural goods $(T=1)$. Recall that the intuition for our result is that with $n>\tilde{n}$ we expect that increased $\gamma$ should have more impact on consumption network externalities for home than foreign goods (see equation 4). But protection of foreign cultural goods ( $T>1)$ can actually yield $n<\tilde{n}$, raising the possibility that the impact of globalization will be reversed and that increased $\gamma$ will raise rather than reduce the relative number of foreign varieties. ${ }^{12}$ With this caveat, we state:

Proposition 4: Given $1 / 2 \leq z<z_{H}(\gamma)$, we obtain $\partial R(z, \gamma, T=1) / \partial \gamma<0$.

Proposition 4 implies that the welfare effects of increased globalization are ambiguous. Higher $\gamma$ implies higher consumption network externalities, unambiguously increasing consumer utility in the current period. However, higher $\gamma$ also reduces the ratio of relatively scarce foreign varieties to relatively abundant home varieties of cultural goods. At the same time, the total number of varieties is fixed given $T=1$ : from equations (7) and (8) we have $n+\widetilde{n}=\left[\alpha\left(L+L^{*}\right)-(T-1)(x-D)\right] / p x=\left[\alpha\left(L+L^{*}\right)\right] / p x$, so the absolute number of foreign varieties must decline as $\gamma$ increases. If foreign varieties are sufficiently scarce initially, the quality of future cultural goods given by equation (3) must fall. Consequently, the welfare of the representative consumer in both countries

\footnotetext{
${ }^{12}$ We have confirmed this possibility using numerical simulations.
} 
could decrease if the rate of time discount is sufficiently small. A fall in welfare of the representative home consumer could be termed "cultural blowback." 13

In addition to predictions regarding the impact of increased globalization, our model can make predictions concerning market shares for cultural goods that differ in the extent to which they are socially consumed. These will be cross-sectional rather than time-series predictions. Propositions 5 and 6 are the analogues of Propositions 1 and 4, respectively.

Proposition 5: Suppose that the assumptions of Proposition 1 hold. Consider two types of cultural goods, 1 and 2, such that $H_{1}(c)>H_{2}(c)$. That is, good 1 has stronger consumption network externalities than good 2. Let us label the solutions for the model in the two cases by the subscripts 1 and 2 . Then the maximum $z$ that is consistent with $\tilde{n}_{1}$ $>0$ is smaller than the maximum $z$ that is consistent with $\tilde{n}_{2}>0$ : production will disappear from a larger foreign country when the cultural goods have stronger consumption network externalities.

Proposition 5 implies that as the externality becomes stronger, the region of cultural diversity shrinks as shown in Figure 1, where we now make use of two cultural diversity frontiers: the outside frontier, labeled $z_{H 2}(\gamma)$, refers to the weaker externality, and the inside frontier, labeled $z_{H 1}(\gamma)$, refers to the stronger externality. This shift of the cultural diversity frontier is useful in the proof of the next proposition, which also requires that $H_{1}(c)-H_{2}(c)$ is not too large, analogous to our standard restriction on $H^{\prime}$.

Proposition 6: Suppose that the assumptions of Proposition 4 hold, including $T=1$. Consider two types of cultural goods, 1 and 2, such that $H_{1}(c)>H_{2}(c)$. Let us label the ratios of $\tilde{n}$ to $n$ in the two cases by $R_{1}(z, \gamma, T=1)$ and $R_{2}(z, \gamma, T=1)$. If $H_{1}(c)-H_{2}(c)$ is not too large, then $R_{1}(z, \gamma, T=1)<R_{2}(z, \gamma, T=1)$.

\footnotetext{
${ }^{13}$ It is tempting to see the current creative travails of Hollywood as evidence that this is already taking place. One critic (Elliott 2005) wrote of the 2005 summer season, "When retreads so dominate the box office, it says Hollywood is open for business in much the same spirit as Forest Lawn - only the dead and recycled seem truly served," and director Terry Gilliam stated, "I know when I watch trailers, that I'm seeing the same ones I've seen the last 15 years....Maybe the public is waking up that they're watching the same film again." We prefer to note cautiously that cultural creativity, like macroeconomic time series, displays cycles as well as trends.
} 
The most promising way to test Propositions 5 or 6 is to look within a category of cultural goods for types that clearly differ in the extent to which the social aspect of consumption is important. This will hold constant as many confounding factors (e.g., comparative advantage and trade costs) as possible. For example, we would predict that the market share of Western clothing would be greater for business attire than for sleepwear. An especially interesting comparison is film versus television. Even primetime television is less viewed and discussed with individuals outside the home than are films, on average. The consensus view among media scholars is that the U.S. share of foreign television viewing, even for primetime, is far below the U.S. share of foreign cinema admissions. ${ }^{14}$ Unfortunately, systematic ratings data giving market shares of U.S. and foreign programming are unavailable or expensive for most countries. We know that the U.S. audience share for the largest commercial network in S. Korea has fluctuated between 5 and 13 percent during the period 1992-2002 (Chung 2003), compared to a 2002 cinema market share of 49 percent in Table 1. An article in Variety (Johnson 2003) notes, "A recent survey by Nielsen Media Research found that 71\% of the top ten programs in 60 countries were locally produced." As an example of the cultural specificity to which local producers cater, the article cites the popularity of "religious fiction" in Italy: "Hardly a week goes by without a spiritual offering in primetime, a series or made-for based on the Bible or the lives of popes and saints. The latest example of religious fiction was dedicated to the saint Maria Goretti and drew 10 million viewers (a 35\% share)."

These cross-sectional predictions are not implied by an explanation of increasing

\footnotetext{
${ }^{14}$ Straubhaar (2002, p. 197) states, "As ratings in many countries reflect, audiences usually tend to prefer local programming when they can get it."
} 
U.S. or Anglo-American cultural market shares over time that is based on falling rates of cultural discount, which helps to distinguish this explanation from our explanation based on consumption network externalities. Another distinction between these two explanations can be seen from equations (10) and (11): increasing $\delta$ increases each country's consumption of the other country's representative variety relative to its own variety equally, whereas increasing $\gamma$ tends to increase the smaller country's consumption of the larger country's representative variety more relative to its own variety, because $n>$ $\tilde{n}$ in the $H$ functions. ${ }^{15}$

In order to increase their cultural goods market shares, countries around the world such as Australia, Canada, France, and S. Korea have turned to protectionist measures such as requiring that a minimum percentage of total domestic screen time or of each broadcaster's programming be domestic content (Hoskins et al. 1997). For the remainder of this section we investigate the effects of the ad valorem tariff imposed by the foreign country on imports of home cultural goods, which can be thought of as the tariff equivalent of a quantitative restriction. As can be seen from equations (10) and (11), an increase in $T$ directly increases the slope of the foreign consumption schedule in Figure 2 and otherwise affects the home and foreign consumption schedules only through the $H$ functions. Given our restrictions on the $H$ functions the dominant effect will be that illustrated by the dashed line in Figure 2, leading to an increase in both $D$ and $\widetilde{D}$. Proposition 7 shows that these increases dominate the direct effect of $T$ in equation (12), so that the number of foreign varieties rises relative to the number of home varieties:

\footnotetext{
${ }^{15}$ We cannot prove that $D^{*} / \widetilde{D}^{*}$ rises relative to $\widetilde{D} / D$ as $\gamma$ increases, but it does in all of our numerical simulations. A similar effect could be obtained if only the foreign (small) country's rate of cultural discount falls. Bala and Van Long (2005) develop a model in which preferences in the small country evolve towards those of the large country over time.
} 
Proposition 7: Given $1 / 2 \leq z<z_{H}(\gamma)$, we obtain $\partial R(z, \gamma, T) / \partial T>0$.

In the current period protection reduces utility of the representative foreign consumer by raising prices of imported cultural goods, and reduces welfare for all consumers by causing the number of varieties available to be below the free-market equilibrium level of $\alpha\left(L+L^{*}\right) / p x .{ }^{16}$ In all future periods, however, the quality of cultural goods may increase, provided that the increase in $R$ dominates the fall in the total number of varieties so that $\tilde{n}$ increases. ${ }^{17}$ If the rate of time discount is sufficiently small, the welfare of the representative consumer in both countries could increase. However, this scenario for positive welfare effects may be too optimistic if, as suggested in the introduction, the foreign cultural goods production that benefits from protection imitates home cultural goods and therefore fails to generate relatively scarce ideas.

\section{Imitative Cultural Production}

In this section, we introduce the possibility of imitative cultural production into our model. Imitative cultural production is defined as the production of cultural goods that share the cultural discount properties of domestic cultural goods but the consumption network externalities of the other country's cultural goods (i.e., they are "compatible" with the other country's cultural goods). Imitative film would be film that adopts the plot conventions and cinematic style of the other country while employing domestic actors and using a domestic setting. Imitative music would be music that adopts the rhythm, harmony, and instrumentation of the other country while employing domestic singers and using lyrics that address domestic concerns and values. The use of the domestic language

\footnotetext{
${ }^{16}$ This effect can only be avoided if the foreign government spends all the tariff revenue it collects on cultural goods.

${ }^{17} \tilde{n}$ increases with $T$ in all of our numerical simulations.
} 
by the actors or singers in imitative cultural goods would be the most important element in maintaining the cultural discount properties of domestic cultural goods, whereas the adoption of core features of the other country's cultural goods would allow imitative films to be part of the same "conversation" as the other country's films and imitative music to be programmed with the other country's music at parties and on the radio. It is important to emphasize that imitative cultural production in no way involves violation of intellectual property laws, and we continue to maintain our assumption that intellectual property rights are fully protected.

There are now potentially two types of cultural goods being produced in each country. For clarity, we will refer to the cultural goods already present in the model of the previous section as "traditional" as opposed to "imitative." We assume that the fixed cost of producing imitative cultural goods is a multiple $\lambda>1$ of the fixed cost of producing traditional cultural goods, reflecting the costs to domestic producers of adopting the unfamiliar features of the other country's cultural goods. ${ }^{18}$

We begin our analysis maintaining the assumption of consumer homogeneity within each country. Moreover, we provisionally assume that in equilibrium imitative cultural production occurs only in the foreign country. Denoting foreign imitative goods by an overbar, we then write home and foreign demands using equations (13) and (14), respectively:

$$
D=\frac{p^{-\sigma} H^{\sigma-1}}{n p^{1-\sigma} H^{\sigma-1}+\bar{n} p^{1-\sigma}(\delta H)^{\sigma-1}+\widetilde{n} p^{1-\sigma}(\delta \widetilde{H})^{\sigma-1}} \alpha L,
$$

\footnotetext{
${ }^{18}$ The marginal costs of producing the physical film or CD are presumably the same for both types of cultural goods, though the marginal costs of imitative cultural goods could also be higher if, for example, it was necessary to hire supporting artists from the other country and pay them royalties.
} 


$$
\begin{aligned}
& \bar{D}=\frac{p^{-\sigma}(\delta H)^{\sigma-1}}{n p^{1-\sigma} H^{\sigma-1}+\bar{n} p^{1-\sigma}(\delta H)^{\sigma-1}+\widetilde{n} p^{1-\sigma}(\delta \widetilde{H})^{\sigma-1}} \alpha L, \\
& \widetilde{D}=\frac{p^{-\sigma}(\delta \widetilde{H})^{\sigma-1}}{n p^{1-\sigma} H^{\sigma-1}+\bar{n} p^{1-\sigma}(\delta H)^{\sigma-1}+\widetilde{n} p^{1-\sigma}(\delta \widetilde{H})^{\sigma-1}} \alpha L, \\
& D^{*}=\frac{(p T)^{-\sigma}\left(\delta H^{*}\right)^{\sigma-1}}{n(p T)^{1-\sigma}\left(\delta H^{*}\right)^{\sigma-1}+\bar{n} p^{1-\sigma} H^{* \sigma-1}+\widetilde{n} p^{1-\sigma} \widetilde{H}^{* \sigma-1}} \alpha L^{*}, \\
& \bar{D}^{*}=\frac{p^{-\sigma} H^{* \sigma-1}}{n(p T)^{1-\sigma}\left(\delta H^{*}\right)^{\sigma-1}+\bar{n} p^{1-\sigma} H^{* \sigma-1}+\widetilde{n} p^{1-\sigma} \widetilde{H}^{* \sigma-1}} \alpha L^{*}, \\
& \widetilde{D}^{*}=\frac{p^{-\sigma} \widetilde{H}^{* \sigma-1}}{n(p T)^{1-\sigma}\left(\delta H^{*}\right)^{\sigma-1}+\bar{n} p^{1-\sigma} H^{* \sigma-1}+\widetilde{n} p^{1-\sigma} \widetilde{H}^{* \sigma-1}} \alpha L^{*},
\end{aligned}
$$

where for simplicity we again assume that the foreign government spends all the tariff revenue it collects on the homogeneous good.

Since foreign imitative goods are compatible with home goods, we write consumption network externalities as follows:

$$
\begin{aligned}
& H \equiv H\left[n\left(D+\gamma D^{*}\right)+\bar{n}\left(\bar{D}+\gamma \bar{D}^{*}\right)\right], \widetilde{H} \equiv H\left[\widetilde{n}\left(\widetilde{D}+\gamma \widetilde{D}^{*}\right)\right], \\
& H^{*} \equiv H\left[n\left(\gamma D+D^{*}\right)+\bar{n}\left(\gamma \bar{D}+\bar{D}^{*}\right)\right], \widetilde{H}^{*} \equiv H\left[\widetilde{n}\left(\gamma \widetilde{D}+\widetilde{D}^{*}\right)\right] .
\end{aligned}
$$

We also need to make a similar change in the equation for cultural goods quality reflecting that imitative goods are perfect substitutes for home goods in its production:

$$
Q_{\tau}=Q\left(n_{\tau-1}+\bar{n}_{\tau-1}, \widetilde{n}_{\tau-1}\right) .
$$

A sufficient condition for existence of foreign imitative production in equilibrium is that entry by foreign producers of imitative goods is possible when the foreign country is completely specialized in traditional production, i.e., in the equilibrium studied in the 
previous section. The condition for successful entry of imitative goods producers is easily shown to be $\bar{D}+\bar{D}^{*} \geq \lambda x$, where $\bar{D}$ and $\bar{D}^{*}$ are evaluated at the equilibrium without imitative production. Since $\widetilde{D}+\widetilde{D}^{*}=x$, this condition can be rewritten as $\bar{D}+\bar{D}^{*} \geq \lambda\left(\widetilde{D}+\widetilde{D}^{*}\right)$. It is then easily shown that this condition must hold if $(H / \widetilde{H})^{\sigma-1} \geq$ $\lambda$ and $\left(H^{*} / \widetilde{H}^{*}\right)^{\sigma-1} \geq \lambda$, where the $H^{\prime}$ s are evaluated at $\bar{n}=0$. Intuitively, it is profitable to introduce imitative production when its consumption network externalities are sufficiently high relative to those of traditional production to offset its higher cost. The home market effect, by causing $n>\tilde{n}$ in equilibrium, tends to support introduction of imitative production by making $H / \widetilde{H}$ and $H^{*} / \widetilde{H}^{*}$ larger, as can be seen from equation (4).

Summarizing, the conditions $(H / \widetilde{H})^{\sigma-1} \geq \lambda$ and $\left(H^{*} / \widetilde{H}^{*}\right)^{\sigma-1} \geq \lambda$ in the equilibrium without imitative production are sufficient to ensure the existence of imitative production in the foreign country. In fact, these conditions are also sufficient, provided $T$ is not too large, to ensure the existence of an equilibrium without any traditional production in the foreign country and without imitative production in the home country:

Proposition 8: If consumers within each country are homogeneous, $(H / \widetilde{H})^{\sigma-1} \geq \lambda$ and $\left(H^{*} / \widetilde{H}^{*}\right)^{\sigma-1} \geq \lambda$ in the equilibrium without imitative production, and $T$ is sufficiently close to one, an equilibrium exists in which the foreign country is completely specialized in imitative production and the home country is completely specialized in traditional production.

The intuition for the first part of Proposition 8 is that the consumption network externalities for imitative relative to traditional goods in the foreign country are obviously greater in an equilibrium with imitative production and without traditional production 
than vice-versa, and there is no consumer heterogeneity within the foreign country to offset this effect by generating a tendency to diversify consumption. For the second part of Proposition 8 we note that home imitative goods would share consumption network externalities with foreign traditional goods, which are already assumed to be dominated by the consumption network externalities of home traditional goods (shared with foreign imitative goods).

Proposition 8 has important consequences for the welfare effects of protection. The reason is that in an equilibrium in which the foreign country is completely specialized in imitative production and the home country is completely specialized in traditional production, the current mix of varieties between home and foreign no longer affects the quality of future cultural production (see equation 16). Only the total number of current varieties matters, and it is easily shown that this cannot be increased by protection. With the ability to increase the quality of future cultural production gone, protection must reduce consumer welfare.

This important point that foreign protection must reduce welfare when imitative foreign production has driven out traditional foreign production does not tell us either the positive or normative effect of protection in the many real world cases where both traditional and imitative foreign production are present. We conjecture that such cases cannot be analyzed with our homogeneous consumer model, i.e., that a country with homogeneous consumers must completely specialize in either traditional or imitative production. Although we have not been able to prove this conjecture, neither have we been able to find a numerical simulation in which it fails. The argument for the conjecture is simple: without consumer heterogeneity, there is nothing to counter the 
self-reinforcing effect of consumption network externalities that tends to drive the economy to a corner solution.

To solve this problem, we now introduce into our model consumer heterogeneity within the foreign country ${ }^{19}$ that parallels the world consumer heterogeneity that exists in the form of cultural discounts. In particular, in the same way as world consumers are divided into two groups with shares of world population $z$ and $1-z$ and each with a cultural discount $\delta$, foreign consumers can be subdivided into two groups with shares of foreign population $\mu$ and $1-\mu$ and each with a "taste discount" $\beta{ }^{20}$ With a less

developed country context in mind, we will assume that "urban" foreign consumers apply their taste discount to cultural goods in the traditional style of their country, whereas "rural" foreign consumers apply their taste discount to goods in the style of the home country, which includes imitative goods. Finally, we assume that taste discounting is weaker than cultural discounting, so that $\beta>\delta$.

Relative to equations (14), the demands for urban and rural foreign consumers must be modified to incorporate these taste discounts. Urban foreign consumers now discount foreign traditional goods by $\beta$, and rural foreign consumers now discount foreign imitative goods by $\beta$ and home goods by $\beta \delta$. The demand functions for urban foreign consumers are now given by:

\footnotetext{
${ }^{19}$ Preference heterogeneity within a country is also important for sustaining diverse cultural production in the model of Francois and van Ypersele (2002).

${ }^{20}$ A parallel subdivision could be introduced for the home (larger) country. However, unlike foreign imitative production, home imitative production would be disadvantaged relative to home traditional production with regard to consumption network externalities. If in addition the share of home consumers who prefer the foreign style is sufficiently small, the home country will not produce imitative goods in equilibrium, just as the world economy will not produce foreign cultural goods if the home country share of world consumers is too large.
} 


$$
\begin{aligned}
& D_{U}^{*}=\frac{(p T)^{-\sigma}\left(\delta H^{*}\right)^{\sigma-1}}{n(p T)^{1-\sigma}\left(\delta H^{*}\right)^{\sigma-1}+\bar{n} p^{1-\sigma} H^{* \sigma-1}+\widetilde{n} p^{1-\sigma}\left(\beta \widetilde{H}^{*}\right)^{\sigma-1}} \alpha \mu L^{*}, \\
& \bar{D}_{U}^{*}=\frac{p^{-\sigma} H^{* \sigma-1}}{n(p T)^{1-\sigma}\left(\delta H^{*}\right)^{\sigma-1}+\bar{n} p^{1-\sigma} H^{* \sigma-1}+\widetilde{n} p^{1-\sigma}\left(\beta \widetilde{H}^{*}\right)^{\sigma-1}} \alpha \mu L^{*}, \\
& \widetilde{D}_{U}^{*}=\frac{p^{-\sigma}\left(\beta \widetilde{H}^{*}\right)^{\sigma-1}}{n(p T)^{1-\sigma}\left(\delta H^{*}\right)^{\sigma-1}+\bar{n} p^{1-\sigma} H^{* \sigma-1}+\widetilde{n} p^{1-\sigma}\left(\beta \widetilde{H}^{*}\right)^{\sigma-1}} \alpha \mu L^{*},
\end{aligned}
$$

and the demand functions for rural foreign consumers are now given by:

$$
\begin{aligned}
& D_{R}^{*}=\frac{(p T)^{-\sigma}\left(\beta \delta H^{*}\right)^{\sigma-1}}{n(p T)^{1-\sigma}\left(\beta \delta H^{*}\right)^{\sigma-1}+\bar{n} p^{1-\sigma}\left(\beta H^{*}\right)^{\sigma-1}+\widetilde{n} p^{1-\sigma} \widetilde{H}^{* \sigma-1}} \alpha(1-\mu) L^{*}, \\
& \bar{D}_{R}^{*}=\frac{p^{-\sigma}\left(\beta H^{*}\right)^{\sigma-1}}{n(p T)^{1-\sigma}\left(\beta \delta H^{*}\right)^{\sigma-1}+\bar{n} p^{1-\sigma}\left(\beta H^{*}\right)^{\sigma-1}+\widetilde{n} p^{1-\sigma} \widetilde{H}^{* \sigma-1}} \alpha(1-\mu) L^{*}, \\
& \widetilde{D}_{R}^{*}=\frac{p^{-\sigma} \widetilde{H}^{* \sigma-1}}{n(p T)^{1-\sigma}\left(\beta \delta H^{*}\right)^{\sigma-1}+\bar{n} p^{1-\sigma}\left(\beta H^{*}\right)^{\sigma-1}+\widetilde{n} p^{1-\sigma} \widetilde{H}^{* \sigma-1}} \alpha(1-\mu) L^{*} .
\end{aligned}
$$

The externality functions are still described by equation (15), provided we define $D^{*} \equiv D_{U}^{*}+D_{R}^{*}, \bar{D}^{*} \equiv \bar{D}_{U}^{*}+\bar{D}_{R}^{*}$, and $\widetilde{D}^{*} \equiv \widetilde{D}_{U}^{*}+\widetilde{D}_{R}^{*}$. Finally, the market clearing conditions for the representative varieties of home, foreign imitative, and foreign traditional cultural goods are, respectively,

$$
\begin{aligned}
& D+D_{U}^{*}+D_{R}^{*}=x, \\
& \bar{D}+\bar{D}_{U}^{*}+\bar{D}_{R}^{*}=\lambda x, \\
& \widetilde{D}+\widetilde{D}_{U}^{*}+\widetilde{D}_{R}^{*}=x .
\end{aligned}
$$

Equations (13) and (17) - (19) give us twelve equations in twelve unknowns.

This model is too complicated to obtain analytical results, but by using intuition provided by the model of section III plus numerical simulations, we can gain an accurate idea of 
how it works. The two issues we will investigate with this model are the impact of increased globalization (a rise in $\gamma$ ) and the impact of increased protection by the foreign (smaller) country (a rise in $T$ ).

In the previous section we showed that, in our model without imitative goods, an increase in $\gamma$ must reduce the ratio of foreign varieties to home varieties of cultural good produced in equilibrium. Since imitative foreign goods share consumption network externalities with home goods in our current model, we expect that increased globalization will reduce the ratio of traditional foreign varieties to imitative foreign varieties when these two types of cultural production coexist in the foreign country. This proves to be true in all our numerical simulations. In fact, the "import substitution effect" of increased imitative production in response to higher $\gamma$ is strong enough that, although the number and share of foreign traditional varieties in all varieties always fall, in some but not all simulations the number and share of home varieties also fall. It is therefore possible in our current model that increased globalization will increase the share of the home style in world cultural goods production yet decrease the share of home varieties.

In our model without imitative goods, we showed that an increase in $T$ must increase the production of foreign varieties relative to home varieties of cultural goods. When imitative and traditional cultural goods production coexist in the foreign country, the impact of protection in stimulating production of traditional foreign varieties is offset by the differential impact of protection on urban versus rural foreign consumers. The representative urban foreign consumer does not have a taste discount for home and imitative foreign varieties and therefore consumes more of them than does the representative rural foreign consumer. As a result urban consumers are more strongly 
affected by the rise in the price of home varieties caused by the increase in $T$, and they will substitute towards domestic varieties more than rural consumers, thereby increasing the demand for imitative relative to traditional foreign varieties. ${ }^{21}$ To illustrate, we compared the impact of the introduction of a ten percent tariff in the model of section III to its impact in the current model. We chose parameters for the model without imitative production so that the increase in the number of (traditional) foreign varieties was 5.0 percent. We then simulated the current model with the same values of all the parameters common to the two models, plus a ten percent fixed cost premium for imitative goods ( $\lambda$ $=1.1)$, an equal division of foreign consumers into urban and rural $(\mu=0.5)$, and a taste discount twenty percentage points smaller than the cultural discount $(\beta=0.4$, compared to $\delta=0.2$ ). The number of traditional foreign varieties increased by only 1.5 percent, compared to an 18.6 percent increase in the number of imitative foreign varieties.

We conclude that when traditional and imitative cultural goods production coexist in the foreign (smaller) country, protection from imports of home cultural goods is less likely to increase welfare by increasing quality of future cultural goods production because most of the boost to foreign cultural production is wasted on imitative varieties. ${ }^{22}$ Because production of foreign traditional varieties may still increase, however, the possibility that protection raises the welfare of all consumers remains.

\footnotetext{
${ }^{21}$ This effect in turn is dampened by the fact that reduced production of home varieties decreases the consumption network externality for imitative foreign varieties.

${ }^{22}$ Mas-Colell (1999) distinguishes between "protection of national cultural production" (protection that attempts "to guarantee that, for example, in the European Union there is a significant production of movies, even if they are, in every other respect, entirely similar to those produced in Hollywood") and "protection of the production of national culture" (in which "the intent is to promote the availability and the consumption of movies transmitting 'Spanish,' 'French,' or 'Catalan' content: language, historical episodes, costumes, traditions").
} 


\section{Conclusions and Lessons for Cultural Policy}

We applied to international trade in cultural goods a model of home market effects with consumption network externalities. Consumption network externalities reinforce the home market effect, the reinforcement being stronger for cultural goods for which consumption network externalities are stronger. Increased sharing of consumption network externalities across countries also exacerbates the home market effect, so increased communication and travel between countries will increase the cultural market share of the larger country. By reducing cultural diversity, this aspect of globalization entails an intertemporal welfare tradeoff if ideas of different cultures are imperfect substitutes in production of future cultural goods quality. Protection of domestic cultural goods production, actually practiced by many of the world's smaller countries, may do little to preserve cultural diversity because it primarily stimulates production of cultural goods that imitate those of the larger country in order to share in their consumption network externalities.

Current cultural policy in many countries is to set aside some percentage of total domestic screen time for domestically produced film and some percentage of total domestic air time for domestically produced music or television. These are quantitative restrictions, surviving the General Agreement on Trade in Services by the unwritten doctrine of "cultural exception" (UNESCO n.d.), for which tariff equivalents can be found (at least in theory). According to our model, it is at least possible that these policies are welfare-improving, because they may be increasing production of traditional cultural goods that generate ideas with large marginal impacts on the quality of future cultural goods output in all countries. However, to the extent that these policies protect 
or even stimulate production of imitative cultural goods, they are purely welfaredecreasing. This negative outcome becomes more likely over time as increased globalization makes imitative production ever more attractive. In any case, standard economic reasoning suggests that import restrictions are an inefficient way to achieve a target level of domestic output. In our model, moreover, replacing import restrictions with production subsidies has the special advantage that production subsidies can be directed towards traditional production, whereas import restrictions cannot.

Our model therefore suggests that cultural policy could be reformed by removing import restrictions and replacing them with production subsidies that maintain at least the pre-existing level of traditional cultural output. Since the purpose of subsidizing production of traditional cultural goods is to make the ideas embedded in them available to producers worldwide, subsidies could be to the fixed costs of production rather than to the marginal costs of production or distribution. The cultural output could then be made available to international film and music festivals at no more than marginal cost. This policy reform thus translates into replacing import restrictions with a combination of increases in the budgets for national arts agencies and retargeting of their existing resources, which sometimes appear to be aimed more at supporting domestic cultural employment than domestic cultural ideas (Economist 1998). The practical difficulty with implementation of this reform, to which we already alluded in our introduction, is that taxpayers in small countries would be explicitly funding programs for which most of the benefits accrue to the rest of the world, simply by virtue of its larger size. This suggests that, in exchange for removing its restrictions on imports 
of cultural goods, a small country could ask the large country (the United States) to contribute to the funding of its national arts agency.

Our discussion so far has assumed that the national arts agencies that administer production subsidies for cultural goods will have no difficulties in distinguishing traditional from imitative production. In practice, explicitly targeting "traditional" cultural goods runs the risk of discouraging innovation, thereby stifling rather than rewarding creativity and thus reducing the quality of the ideas that are embedded in the output. ${ }^{23}$ Insofar as imitative cultural production also tends to be "imitative" in the sense of lacking originality, subsidies could be awarded on the basis of artistic merit, thereby erring on the side of allowing some imitative production to be subsidized rather than risk encouraging stagnation. This puts the appropriate criteria for national arts agencies in the hands of the cultural goods producers themselves, who hold the real expertise in this area.

\footnotetext{
${ }^{23}$ As its first definition of "tradition," The American Heritage Dictionary, Second College Edition states, "The passing down of elements of a culture from generation to generation." "Traditional" cultural goods production then preserves those "elements of a culture" that make the embedded ideas imperfect substitutes for the ideas embedded in the dominant cultural output. At the same time, Webster's College Thesaurus gives "fixed" as one of the synonyms for "traditional."
} 


\section{REFERENCES}

Bala, Venkatesh and Long, Ngo Van. 2005. "International Trade and Cultural Diversity with Preference Selection.” European Journal of Political Economy 21(1): 143 162.

Baxter, John. 1999. Mythmaker: The Life and Work of George Lucas (New York: Avon).

Chung, Yoonkyung. 2003. "Television Imports and Consumption." Korean Broadcasting Institute (December), processed.

Church, Jeffrey and Gandal, Neil. 1992. "Network Effects, Software Provision and Standardization.” Journal of Industrial Economics 40(1): 85-103.

Cowen, Tyler. 2002. Creative Destruction: How Globalization is Changing the World's Cultures (Princeton and Oxford: Princeton University Press).

Eaton, B. Curtis; Pendakur, Krishna; and Reed, Clyde G. 2003. "Socializing, Shared Experience, and Popular Culture." University of Calgary Discussion Paper, http://econ.ucalgary.ca/fac-files/bce/culture9.pdf.

The Economist. 1998. "Culture Wars.” September 12, pp. 99-103.

Elliott, David. 2005. "I Know What You Didn't Do Last Summer." San Diego UnionTribune, September 11, pp. F1, F8.

Evans, David. 2002. "The Development of the Blues." In Allan Moore, ed., The Cambridge Companion to Blues and Gospel Music (Cambridge: Cambridge University Press).

Everett, Walter. 1999. The Beatles as Musicians: Revolver through the Anthology (New York: Oxford University Press).

Falicov, Tamara L. 2000. "Argentina's Blockbuster Movies and the Politics of Culture under Neoliberalism, 1989-1998." Media, Culture, and Society 22(3): 327-342.

Francois, Patrick and van Ypersele, Tanguy. 2002. "On the Protection of Cultural Goods.” Journal of International Economics 56(2): 359 - 369.

Grossman, Gene and Helpman, Elhanan. 1991. Innovation and Growth in the Global Economy (Cambridge, MA: MIT).

Helpman, Elhanan and Krugman, Paul. 1985. Market Structure and Foreign Trade (Cambridge, MA: MIT). 
Hirsch, Foster. 2001. The Dark Side of the Screen: Film Noir (Da Capo Press).

Hoskins, Colin; McFadyen, Stuart; and Finn, Adam. 1997. Global Television and Film: An Introduction to the Economics of the Business (Oxford: Oxford University Press).

Janeba, Eckhard. 2004. "International Trade and Cultural Identity." National Bureau of Economic Research Working Paper No. 10426 (April).

Johnson, Debra. 2003. “Auds Prefer Local Flavor Over U.S. Fare.” Variety, March 24, p. A3.

Mas-Colell, Andreu. 1999. "Should Cultural Goods Be Treated Differently?" Journal of Cultural Economics 23: 87 - 93.

Ottaviano, Gianmarco and Peri, Giovanni. 2004. "The Economic Value of Cultural Diversity: Evidence from U.S. Cities." National Bureau of Economic Research Working Paper No. 10904 (November).

Pavcnik, Nina. 2002. "Trade Disputes in the Commercial Aircraft Industry." World Economy 25 (May): 733-751.

Romer, Paul M. 1990. “Endogenous Technological Change.” Journal of Political Economy 98(5): S71-102.

Slonimsky, Nicolas. 1997. Baker’s Dictionary of Music (New York: Schirmer).

Straubhaar, Joseph. 2002. “(Re)asserting National Television and National Identity Against the Global, Regional and Local Levels of World Television." In Joseph M. Chan and Bryce T. McIntyre, eds., In Search of Boundaries: Communication, Nation-States, and Cultural Identities (Westport, CT: Ablex).

Stuessy, Joe. 1990. Rock and roll: Its History and Stylistic Development (Englewood Cliffs, NJ: Prentice-Hall).

Thomas, Rosie. 1985. "Indian Cinema: Pleasures and Popularity." Screen 26(3-4): 116-131.

UNESCO. n. d. http://www.unesco.org/culture/industries/trade/html_eng/question17.shtml\#17. 
Table 1

U.S. \& National Cinema Market Shares

\begin{tabular}{lcccc}
\hline & \multicolumn{2}{c}{1985} & \multicolumn{2}{c}{2002} \\
& U.S. & National & U.S. & National \\
\hline France & 39 & 44 & 56 & 34 \\
Germany & 59 & 23 & 83 & 12 \\
Italy & 49 & 32 & 63 & 22 \\
South Korea & & & 49 & 45 \\
Thailand & & & 75 & 23 \\
\hline
\end{tabular}

Notes: Market shares for Thailand are based on gross box office receipts, while all other countries' market shares are based on total admissions. Shares from 1985 are the earliest available figures for France, Germany and Italy. No earlier observations are available for South Korea and Thailand.

Sources : European Audiovisual Observatory; Screen Digest; Spitzenorganisation der Filmwirtschaft; Variety Magazine. 


\section{APPENDIX}

\section{Proof of Proposition 1}

First, note that $L=y z$ and $L^{*}=y(1-z)$. Define the function $z_{H}(\gamma)$ as the lowest $z$ that makes $\tilde{n}=0$. From equation (9), $\tilde{n}=0$ implies $D=T z x /(T z+1-z)$. Furthermore, equation (7) implies $n=\alpha y(T z+1-z) / T x$. If we substitute these two equalities and $\tilde{n}=0$ into equations (10) and (11) and add the latter two equations, we get an equation for $Z_{H}(\gamma)$ :

$$
f(z) \equiv \frac{T z \delta^{\sigma-1} /(T z+1-z)}{h^{\sigma-1}}+\frac{(1-z) /(T z+1-z)}{\delta^{\sigma-1} h^{* \sigma-1}}=1,
$$

where the first equality defines the auxiliary function $f($.$) , and we use the simplifying$ definitions: $h=H[\alpha y(T z+\gamma(1-z)) / T]$ and $h^{*}=H[\alpha y(\gamma T z+1-z) / T]$. It is easily seen that $f(0)>1$ and $f(1)<1$, and that in the limit where the derivatives of $H($.$) are$ sufficiently small the function $f(z)$ must be decreasing, since that is certainly true when $H()=$. constant. Therefore $f(z)$ will pass through one exactly once, and $z_{H}(\gamma)$ is defined the solution of equation (A1): $f\left(z_{H}(\gamma)\right)=1$.

Next, we investigate the first value of $z$ (from above) that makes $n=0$. Without going through the details, we obtain a condition that defines this lower bound for $z$ :

$$
g(z) \equiv \frac{z}{\delta^{\sigma-1} \widetilde{h}^{\sigma-1}}+\frac{(1-z) \delta^{\sigma-1}}{T^{\sigma} \widetilde{h}^{* \sigma-1}}=1,
$$

where we define the new auxiliary function $g($.$) , and define \widetilde{h}=H[\alpha y(z+\gamma(1-z))]$ and $\widetilde{h}^{*}=H[\alpha y(\gamma z+1-z)]$. Note that $g(0)<1$ and $g(1)>1$, and $g(z)$ is increasing in the limit of slowly varying $H$. Again, $g(z)$ passes through one exactly once, and we call $z_{F}(\gamma)$ (the solution of equation (A2)) the maximum $z$ that makes the foreign country the only producer of the cultural good.

We need $z_{H}(\gamma)>1 / 2$ and $z_{F}(\gamma)<1 / 2$. It is therefore sufficient that $f(1 / 2)>1$ and $g(1 / 2)>1$, or

$$
\begin{aligned}
& \frac{T \delta^{\sigma-1} /(T+1)}{h^{\sigma-1}}+\frac{1 /(T+1)}{\delta^{\sigma-1} h^{*-1}}>1, \text { and } \\
& \frac{1}{2 \delta^{\sigma-1} \widetilde{h}^{\sigma-1}}+\frac{\delta^{\sigma-1}}{2 T^{\sigma} \widetilde{h}^{* \sigma-1}}>1 .
\end{aligned}
$$

The second inequality is guaranteed if $\delta$ is sufficiently small and $H$ has an upper bound that is independent of $\delta$. The first inequality must hold if these same two conditions are met and $T$ is finite.

Finally, it is clear from equation (A1) that the denominators of $f(z)$ are increasing in $\gamma$, so increasing $\gamma$ shifts down $f(z)$. Since $f(z)$ is decreasing in $z$, it follows that $z_{H}(\gamma)$ satisfying $f\left(z_{H}(\gamma)\right)=1$ decreases with $\gamma$. 


\section{Proof of Proposition 2}

Since both countries produce the cultural good, the derivation of equations (10) and (11) is valid. Furthermore, under the restrictions that $H($.) has a sufficiently small derivative and that $H$ is bounded from above by $1 / \delta$, the plots of equations (10) and (11) must cross as in Figure 2, establishing the existence of unique equilibrium values of $D$ and $\widetilde{D}$ and therefore of $D^{*}=x-D$ and $\widetilde{D}^{*}=x-\widetilde{D}$. These solutions can be substituted into equations (9) to obtain unique equilibrium values of $n$ and $\tilde{n}$, which must be positive given the assumption that the economy is in the region of cultural diversity.

\section{Proof of Proposition 3}

Suppose that $z$ increases, keeping $y$ fixed, that is, $L$ increases while $L+L^{*}$ remains constant. Then it is easy to see from equations (9) that the direct effect of this change is to increase $n$ and decrease $\tilde{n}$. Furthermore, the indirect impact on $n$ and $\tilde{n}$ through any changes in $D$ and $\widetilde{D}$ can be seen from equations (10) and (11) to occur through the $H$ functions only, and is therefore dominated in first order by the direct effect.

\section{Proof of Proposition 4}

Define (with slight abuse of notation) the function $R(z, \gamma) \equiv R(z, \gamma, T=1)$. Let us plot $R(z, \gamma)$ on top of the region of interest (the region of cultural diversity in Figure 1). We are interested in characterizing the shape of this plot. First, it is easy to see that $R(z, \gamma)$ is identically one along the left edge of the region (where $z=1 / 2$ ). Indeed, when $z=1 / 2$ and $T=1$, the two countries are in all respects identical and symmetry implies that $D=\widetilde{D}^{*}=x-\widetilde{D}$. Substituting $z=1 / 2$ and $\widetilde{D}=x-D$ into equation (12), it is trivial to check that $R(z=1 / 2, \gamma)=1$, regardless of the value of $\gamma$. Also, by construction $R(z, \gamma)$ is zero along the right edge of the region (where $z=z_{H}(\gamma)$ ). Thus, $R(z, \gamma)$ has the shape of half of a car hood, as seen from the left to the right.

Now cut the surface $R(z, \gamma)$ just described with two vertical planes defined by having constant but slightly different values of $\gamma$. The first plane has $\gamma=\bar{\gamma}$, the second has $\gamma=\bar{\gamma}+d \bar{\gamma}$. The intersections between the two planes and the surface $R(z, \gamma)$ would be given by the two curves in Figure 3, labeled $R(z, \bar{\gamma})$ and $R(z, \bar{\gamma}+d \bar{\gamma})$, respectively (ignore for now the labels $R_{1}(z, \gamma)$ and $R_{2}(z, \gamma)$ ). As noted, both curves must start at one, and must end at zero at $z=z_{H}(\bar{\gamma})$ and $z=z_{H}(\bar{\gamma}+d \bar{\gamma})$, respectively. Proposition 1 states that $z_{H}(\bar{\gamma})>z_{H}(\bar{\gamma}+d \bar{\gamma})$. Given these characteristics of the two curves, the simplest possible way to draw them would be as in case I of Figure 3, which would immediately imply the result: $\partial R(z, \bar{\gamma}) / \partial \gamma \leq 0$. Consider, however, the possibility depicted in case II. There, in some regions, $\partial R(z, \bar{\gamma}) / \partial \gamma>0$. It must also be that $R(z, \bar{\gamma})$ and $R(z, \bar{\gamma}+d \bar{\gamma})$ cross each other at an interior point. We need to argue that case II (along with more complicated cases where the curves cross more than once) is not possible. 
In order to do so, we plot in Figure 4 the implied shapes for $\partial R(z, \bar{\gamma}) / \partial \gamma$ as a function of $z$, for both case I and case II. Note that we can actually calculate $\partial R(z, \bar{\gamma}) / \partial \gamma$, and that will allow us to see that case II cannot occur when the derivatives of the $H$ functions are sufficiently small. We begin by totally differentiating equations (10) and (11) with respect to $\gamma$, dropping any terms with higher order derivatives of the $A$ and $B$ functions whenever they add to terms with lower order derivatives (recall that the $A$ and $B$ functions are just combinations of the $H$ functions). After solving for $d D / d \gamma$ and $d \widetilde{D} / d \gamma$, this yields:

$$
\begin{aligned}
& \frac{d D}{d \gamma}=\frac{D \frac{\partial A}{\partial \gamma}+(x-D) \frac{\partial B}{\partial \gamma}}{B-A}, \\
& \frac{d \widetilde{D}}{d \gamma}=\frac{D B \frac{\partial A}{\partial \gamma}+(x-D) A \frac{\partial B}{\partial \gamma}}{B-A} .
\end{aligned}
$$

Next, we insert these equalities into $\partial R(z, \bar{\gamma}) / \partial \gamma$ (which can be easily calculated from equation 12), and simplify the resulting expression with the aid of equations (10) and (11). The end result of this procedure is:

$$
\frac{\partial R(z, \bar{\gamma})}{\partial \gamma}=\frac{\frac{\partial A}{\partial \gamma} D x(B-1)(1-z)+\frac{\partial B}{\partial \gamma}(x-D) x(1-A) z}{(B-A)(\widetilde{D}-x z)^{2}} .
$$

We cannot sign this expression directly, but we can examine how it changes with $z$. If this expression were to change with $z$ as in Figure 4, case II, then its numerator alone would also have the same type of shape, given that the denominator is positive everywhere (note that $B>1>A$ ). But when the derivatives of the $H$ functions are sufficiently small, the numerator approaches a linear function of $z$ (recall that $D$ depends on $z$ only through $A$ and $B$, as can be seen from equations 10 and 11). Therefore, the numerator cannot approximate any shape like Figure 4, case II, while it can approximate Figure 4, case I. This rules out not only case II, but even more complicated cases where $\partial R(z, \bar{\gamma}) / \partial \gamma$ crosses the horizontal axis more than once.

\section{Proof of Proposition 5}

Define $z_{H 1}(\gamma)\left(z_{H_{2}}(\gamma)\right)$ as in Proposition 1 to be the lowest $z$ that makes $\tilde{n}=0$ for a given $\gamma$, in the case when the consumption externality is given by $H_{1}(c)\left(H_{2}(c)\right)$. We want to prove that $Z_{H 1}(\gamma)<Z_{H 2}(\gamma)$.

Note that both $Z_{H 1}(\gamma)$ and $Z_{H_{2}}(\gamma)$ are defined by equations similar to equation (A1). Denote the left-hand side of these equations by $f_{1}(z)$ and $f_{2}(z)$, respectively. Given $H_{2}(c)<H_{1}(c)$, it is clear from equation (A1) that $f_{2}(z)$ must lie above $f_{1}(z)$. Since $f(z)$ decreases with $z$, it follows that $z_{H_{1}}(\gamma)$ satisfying $f_{1}(z)=1$ is less than $z_{H_{2}}(\gamma)$ satisfying $f_{2}(z)=1$. 


\section{Proof of Proposition 6}

The proof is analogous in all respects to proposition 4, and a heuristic argument should suffice. Let us denote with a $\Delta$ the change in any variable when the consumption network externality changes from $H_{1}(c)$ to $H_{2}(c)$, for example, $\Delta D=D_{2}-D_{1}$. We want to prove that the ratio $R(z, \gamma)=\tilde{n} / n$ goes up, that is, $\Delta R(z, \gamma)>0$. In order to calculate $\Delta R(z, \gamma)$ we can use a procedure analogous in all respects to the proof of proposition 4 . The end result is obtained from equation (A3) by replacing all derivatives with respect to $\gamma$ with $\Delta$ :

$$
\Delta R(z, \bar{\gamma})=\frac{\Delta A D x(B-1)(1-z)+\Delta B(x-D) x(1-A) z}{(B-A)(\widetilde{D}-x z)^{2}} .
$$

Note that for the expression above to be valid, the change in the $H$ functions needs to be sufficiently small in exactly the same sense that $\partial H / \partial \gamma$ needed to be small in Proposition 4. Hence the need for the assumption that $H_{1}(c)>H_{2}(c)$ but $H_{1}(c)-H_{2}(c)$ is not too large.

We can now slice the surfaces of $R_{1}(z, \gamma)=\tilde{n}_{1} / n_{1}$ and $R_{2}(z, \gamma)=\tilde{n}_{2} / n_{2}$ with a single vertical surface of constant $\gamma=\bar{\gamma}$. First note that $R_{1}(z=1 / 2, \gamma)=R_{2}(z=1 / 2, \gamma)=1$ (recall that if $T=1$ and $z=1 / 2$, then the model is symmetric across the two countries, therefore $\tilde{n} / n$ must equal one). Furthermore, both $R_{1}(z, \gamma)$ and $R_{2}(z, \gamma)$ end at zero on their respective frontiers. Therefore, we again obtain curves such as depicted in Figure 3, except that now they are labeled as $R_{1}(z, \gamma)$ and $R_{2}(z, \gamma)$, respectively, and we use the result in Proposition 5 that $Z_{H_{1}}(\gamma)<Z_{H_{2}}(\gamma)$. If case I in the figure obtains, then the proof is complete: evidently, $\Delta R(z, \gamma)>0$. If other cases obtain, they would require $\Delta R(z, \gamma)$ as a function of $z$ to be too non-linear to be approximated by the function deduced above.

\section{Proof of Proposition 7}

We can substitute for $T(x-D)$ from equation (11) to obtain from equation (12):

$$
R(z, \gamma, T)=\frac{\widetilde{n}}{n}=\frac{D L^{*}-L \frac{x-\widetilde{D}}{B T^{\sigma-1}}}{L x-\widetilde{D}\left(L+L^{*}\right)} .
$$

Both the numerator and the denominator of this expression are positive in the region of cultural diversity (where both $n>0$ and $\tilde{n}>0$ ). Furthermore, we argued with the aid of Figure 2 that increasing $T$ will increase both $D$ and $\widetilde{D}$, both of which will cause $\tilde{n} / n$ to increase in equation (A4). It is also apparent that the direct effect of the increase in $T$ is to increase $\tilde{n} / n$ in equation (A4). Finally, we can ignore any effect through the function $B$, since $B(D, \widetilde{D}, y, z, \gamma) \equiv\left(\widetilde{H}^{*} / \delta H^{*}\right)^{\sigma-1}$ and therefore changes through $B$ are dominated in first order by the direct effects considered here.

\section{Proof of Proposition 8}

Consider the hypothetical equilibrium in which $\tilde{n}=0$ and $\bar{n}>0$. For this to be an equilibrium, we need $\left.\widetilde{D}\right|_{\tilde{n}=0}+\left.\widetilde{D}^{*}\right|_{\tilde{n}=0}<x$ and $\left.\bar{D}\right|_{\tilde{n}=0}+\left.\bar{D}^{*}\right|_{\tilde{n}=0}=\lambda x$, or $\left.\bar{D}\right|_{\tilde{n}=0}+\left.\bar{D}^{*}\right|_{\tilde{n}=0}>\lambda\left(\left.\widetilde{D}\right|_{\tilde{n}=0}+\left.\widetilde{D}^{*}\right|_{\tilde{n}=0}\right)$. Inspection of equations (13) and (14) reveal that for 
the latter inequality to hold it is sufficient that $\left.H^{\sigma-1}\right|_{\widetilde{n}=0}>\left.\lambda \widetilde{H}^{\sigma-1}\right|_{\tilde{n}=0}$ and $\left.H^{* \sigma-1}\right|_{\tilde{n}=0}>\left.\lambda \widetilde{H}^{* \sigma-1}\right|_{\tilde{n}=0}$. Since when $\tilde{n}=0$, both $\widetilde{H}$ and $\widetilde{H}^{*}$ are identical to one, we can write these sufficient conditions as:

$$
\begin{gathered}
\left.H^{\sigma-1}\right|_{\tilde{n}=0}>\lambda \\
\left.H^{* \sigma-1}\right|_{\tilde{n}=0}>\lambda .
\end{gathered}
$$

Our goal is therefore to prove inequalities (A5). Let us first show that

$$
\left.H^{\sigma-1}\right|_{\tilde{n}=0} \geq\left. H^{\sigma-1}\right|_{\bar{n}=0},
$$

i.e., that the consumption network externality for home and imitative foreign goods is greater than in the equilibrium without imitative production. By definition of the $H$ functions, this is equivalent to showing that

$$
\left[n\left(D+\gamma D^{*}\right)+\bar{n}\left(\bar{D}+\gamma \bar{D}^{*}\right)\right]_{\tilde{n}=0} \geq\left[n\left(D+\gamma D^{*}\right)\right]_{\bar{n}=0} \text {. }
$$

The accounting identity that the representative home consumer uses up his income (analogous to equation 7, for the case with imitative goods) implies $[n D+\bar{n} \bar{D}]_{\tilde{n}=0}=\alpha L / p$. The corresponding accounting identity for the foreign consumer (analogous to equation 8) implies $\left[n D^{*}+\bar{n} \bar{D}^{*}\right]_{\tilde{n}=0}=\alpha L^{*} / p-\left[n(T-1) D^{*}\right]_{\tilde{n}=0}$. Equations (7) and (8) still hold exactly in the equilibrium without imitative production, yielding: $[n D]_{\bar{n}=0}=\alpha L / p-[\widetilde{n} \widetilde{D}]_{\bar{n}=0}$ and $\left[n D^{*}\right]_{\bar{n}=0}=\alpha L^{*} / p-\left[\widetilde{n} \widetilde{D}^{*}\right]_{\bar{n}=0}-\left[n(T-1) D^{*}\right]_{\bar{n}=0}$. Inserting all of these accounting identities into (A7), we can easily show that a sufficient condition for (A7) to hold is $\left[n(T-1) D^{*}\right]_{\tilde{n}=0} \leq\left[n(T-1) D^{*}\right]_{\bar{n}=0}+\left[\widetilde{n} \widetilde{D}^{*}\right]_{\bar{n}=0}$. This must be true when $T=1$ or, by continuity, for $T$ close enough to one.

This establishes inequality (A6). We can prove the first of inequalities (A5) through the following chain of inequalities: $\left.H^{\sigma-1}\right|_{\tilde{n}=0} \geq\left. H^{\sigma-1}\right|_{\bar{n}=0}>\left.\lambda \widetilde{H}^{\sigma-1}\right|_{\bar{n}=0} \geq \lambda$, where the first inequality is inequality (A6), the second inequality comes from the statement of the proposition, and the third inequality follows from the restrictions on the $H$ functions and $\sigma$ that we have assumed throughout the paper. The second of inequalities (A5) is proved in an analogous manner.

We also need to show that, for the equilibrium in which $\tilde{n}=0$ and $\bar{n}>0$, the home country can remain specialized in traditional production. Denoting the hypothetical home imitative goods with an underbar, an equilibrium with $\underline{n}=0$ exists if $\left.\underline{D}\right|_{\tilde{n}=0}+\left.\underline{D}^{*}\right|_{\tilde{n}=0}<\lambda x=\lambda\left(\left.D\right|_{\tilde{n}=0}+\left.D^{*}\right|_{\tilde{n}=0}\right)$. This follows immediately since the hypothetical home imitative goods share the consumption network externalities of foreign traditional goods and $\left.\lambda H\right|_{\tilde{n}=0}>\left.\widetilde{H}\right|_{\tilde{n}=0}=1$ and $\left.\lambda H^{*}\right|_{\tilde{n}=0}>\left.\widetilde{H}^{*}\right|_{\tilde{n}=0}=1$ 


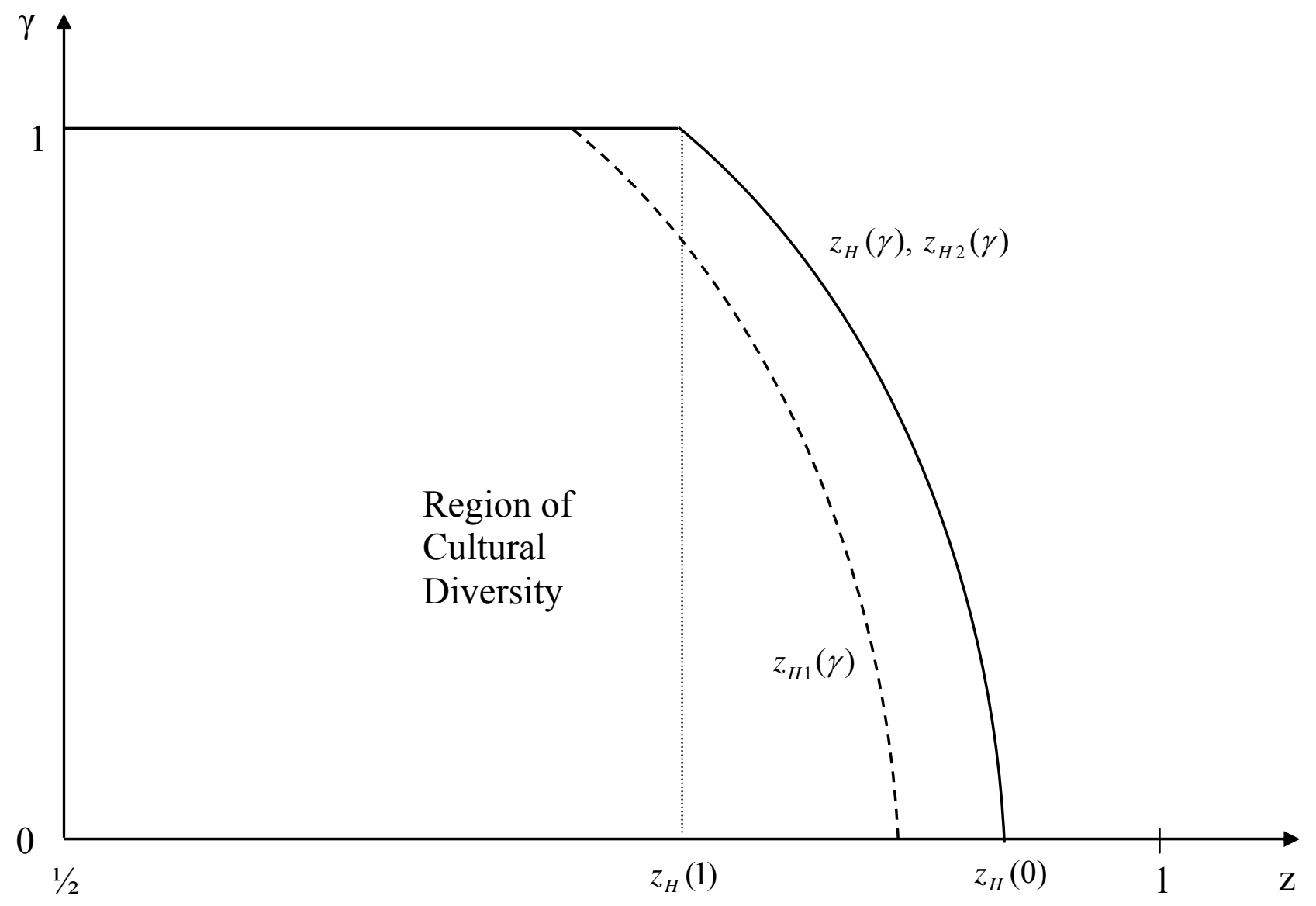

Figure 1 


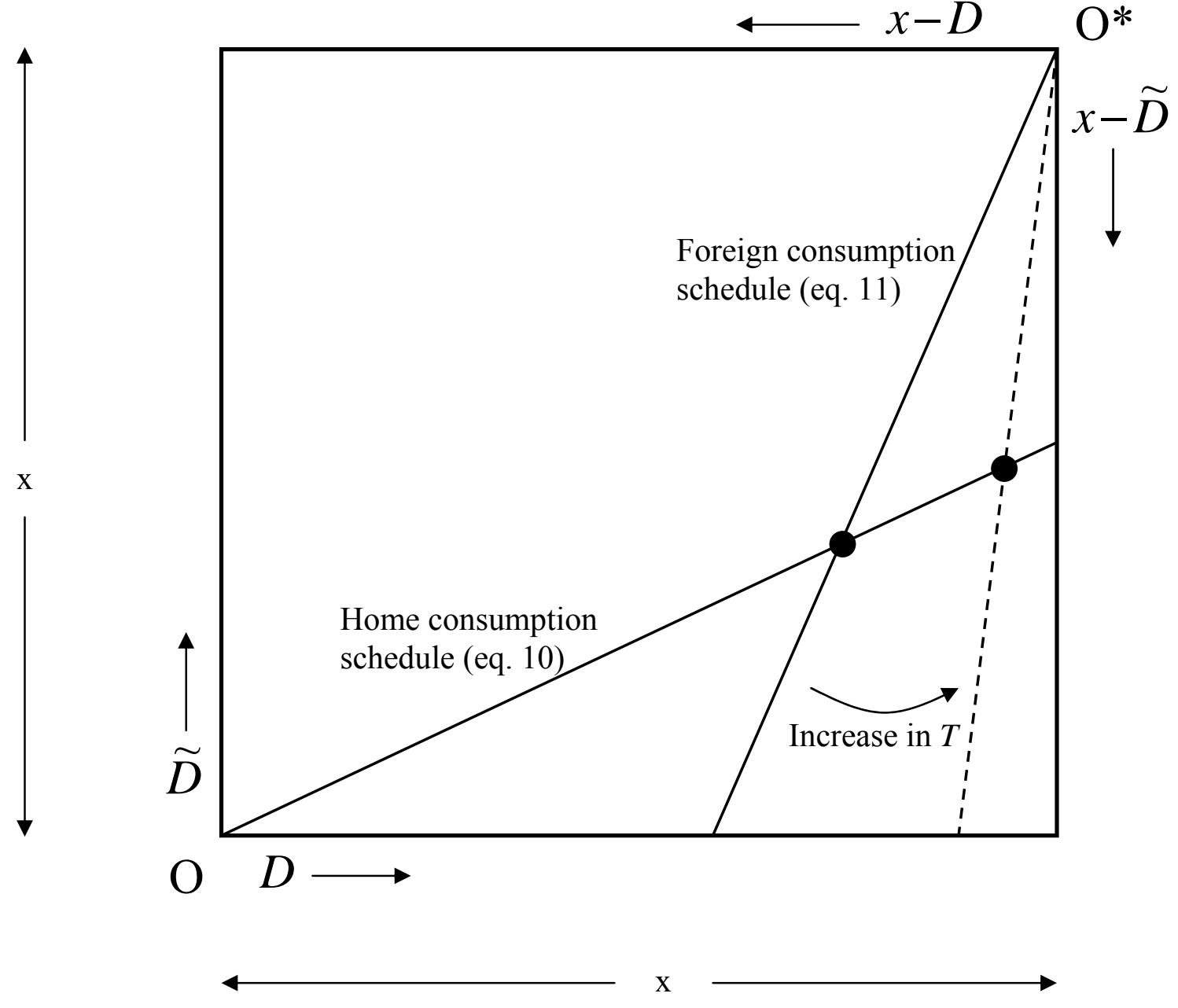

Figure 2 


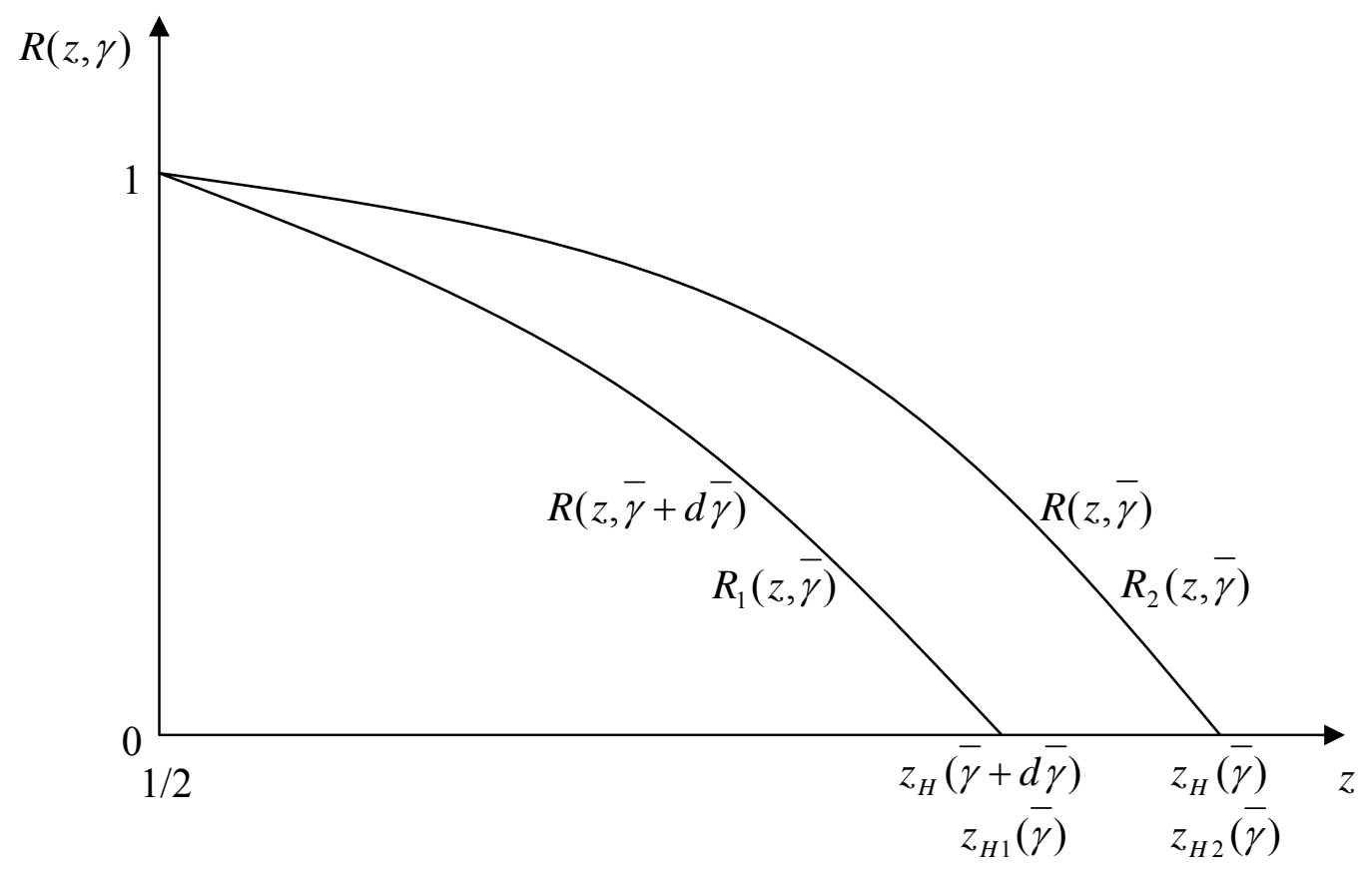

\section{Case I}

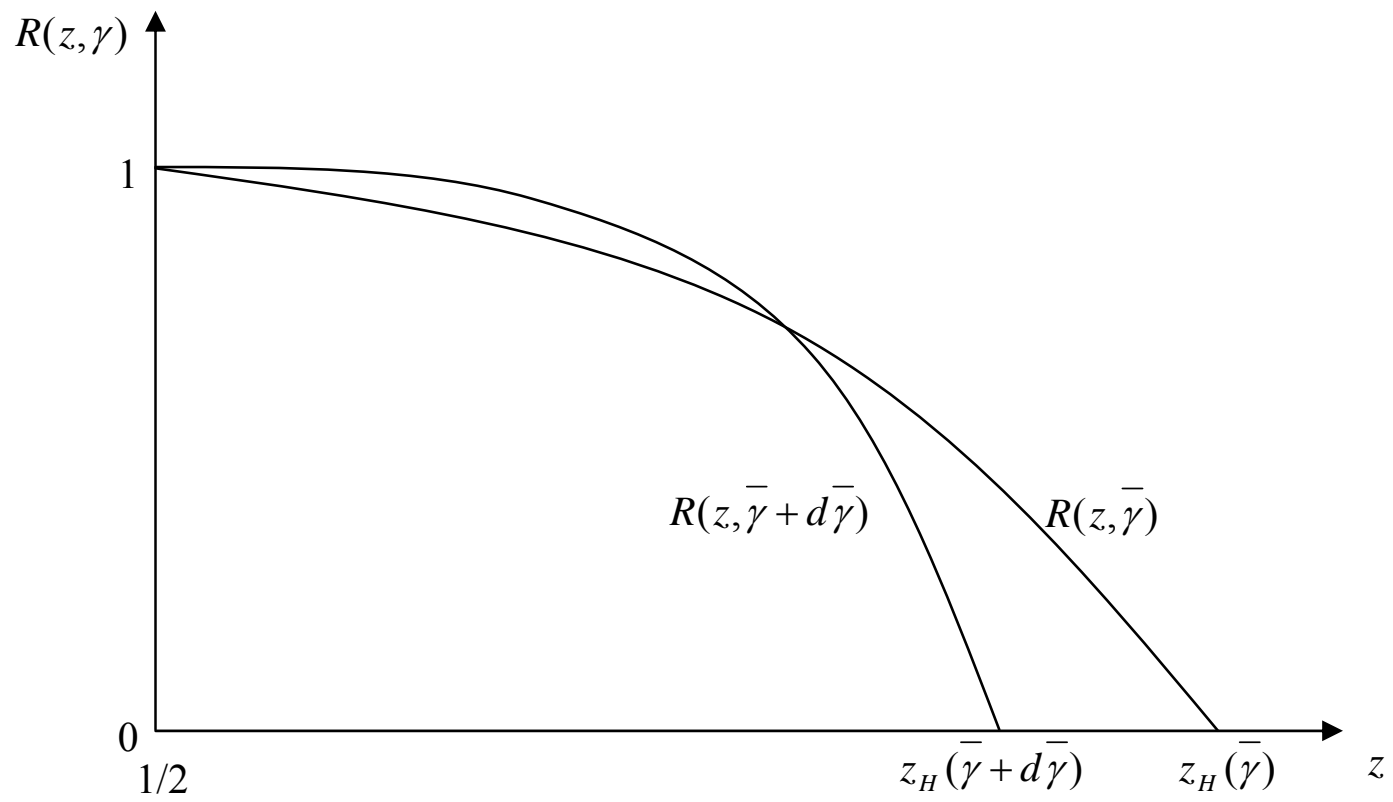

\section{Case II}

Figure 3 


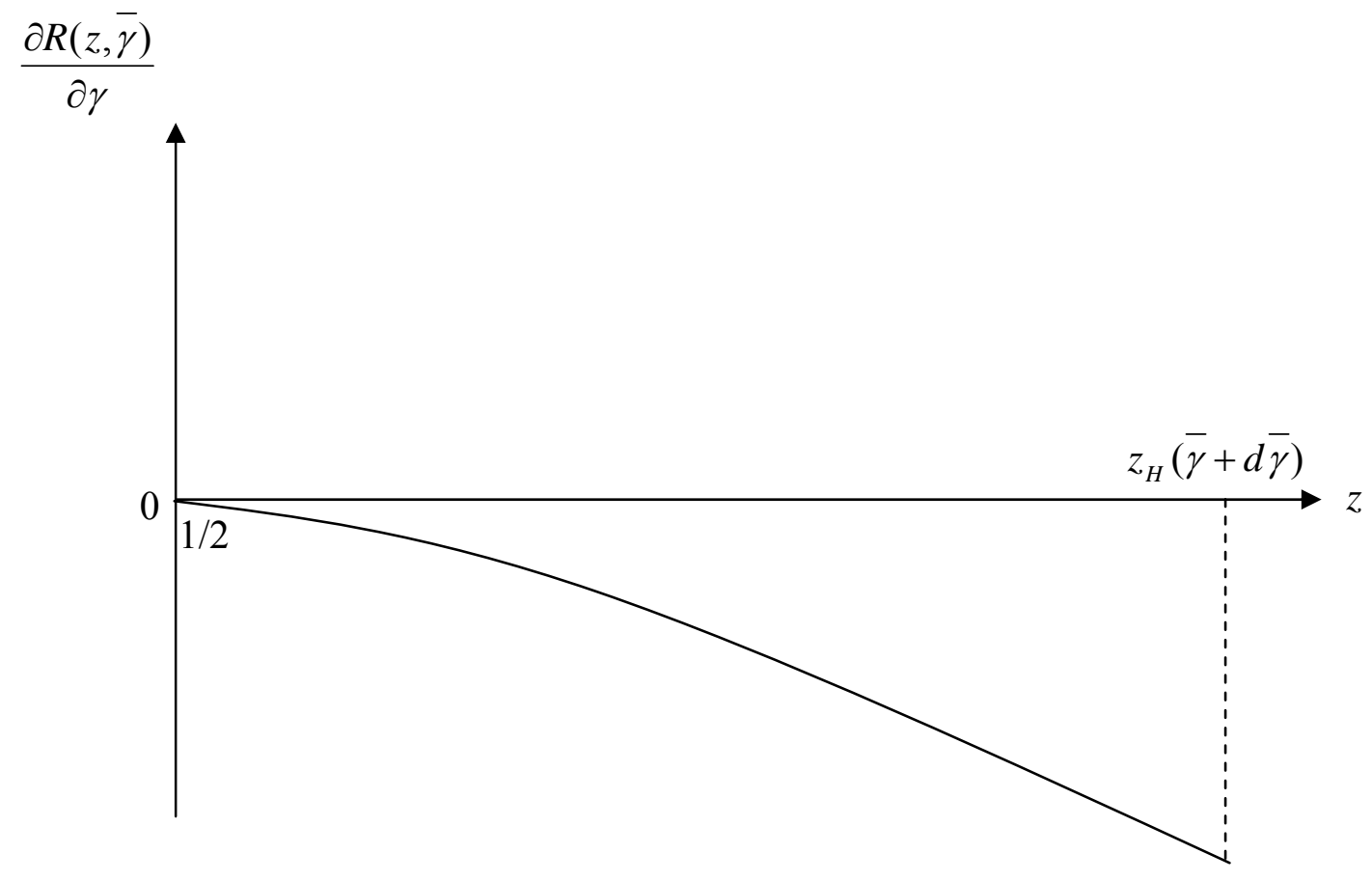

\section{Case I}

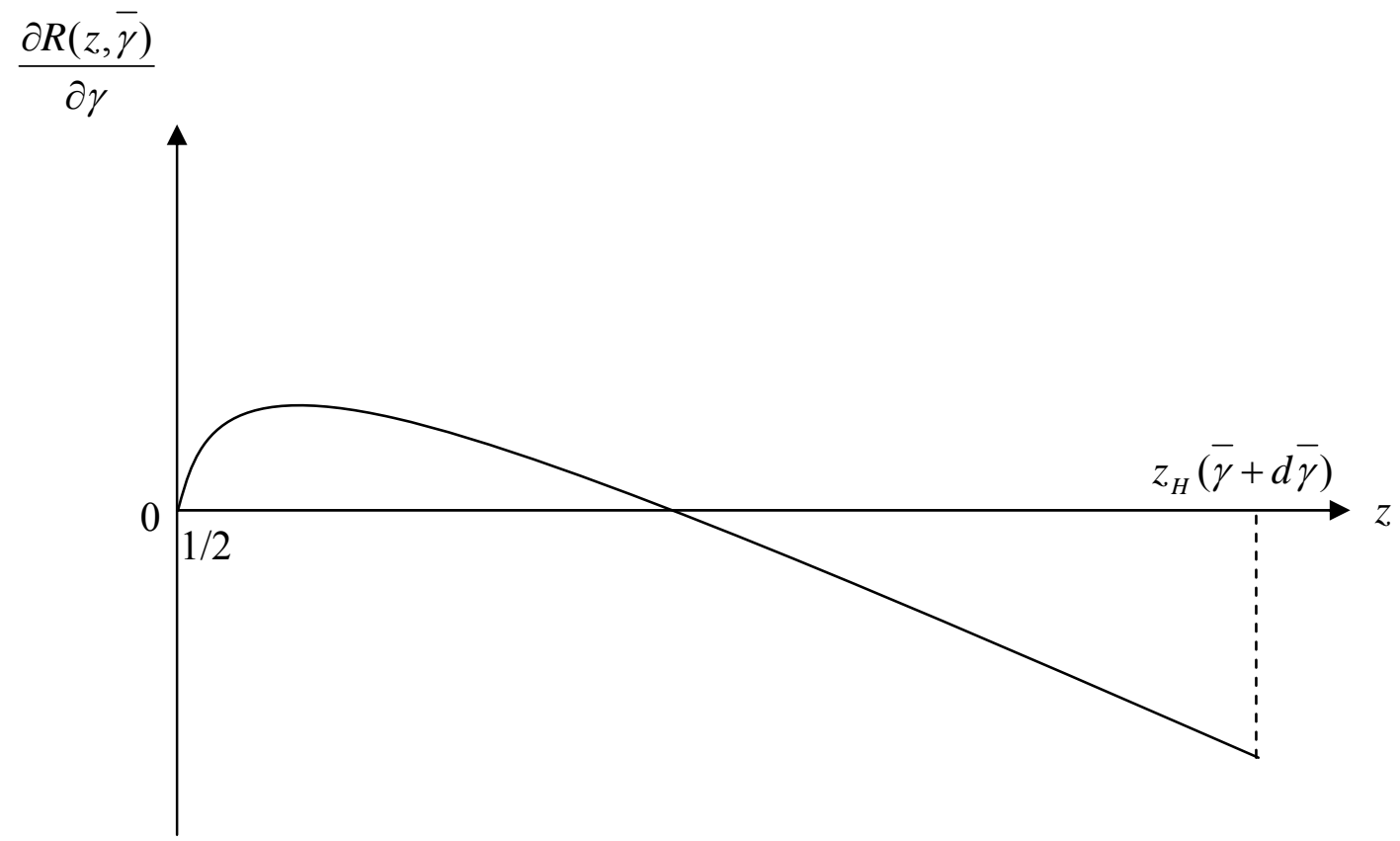

Case II

Figure 4 